\title{
Resonant line transfer in a fog: using Lyman-alpha to probe tiny structures in atomic gas ${ }^{\star}$
}

\author{
Max Gronke ${ }^{1}$, Mark Dijkstra ${ }^{1}$, Michael McCourt ${ }^{2, \star \star}$, and S. Peng $\mathrm{Oh}^{2}$ \\ ${ }^{1}$ Institute of Theoretical Astrophysics, University of Oslo, Postboks 1029, 0315 Oslo, Norway \\ e-mail: maxbg@astro.uio.no \\ 2 Department of Physics, University of California, Santa Barbara, CA 93106, USA
}

Received 20 April 2017 / Accepted 19 July 2017

\begin{abstract}
Motivated by observational and theoretical work that suggest very small-scale $(\lesssim 1 \mathrm{pc})$ structure in the circumgalactic medium of galaxies and in other environments, we study Lyman- $\alpha$ (Ly $\alpha)$ radiative transfer in an extremely clumpy medium with many clouds of neutral gas along the line of sight. While previous studies have typically considered radiative transfer through sightlines intercepting $\lesssim 10$ clumps, we explored the limit of a very large number of clumps per sightline (up to $f_{\mathrm{c}} \sim 1000$ ). Our main finding is that, for covering factors greater than some critical threshold, a multiphase medium behaves similarly to a homogeneous medium in terms of the emergent Ly $\alpha$ spectrum. The value of this threshold depends on both the clump column density and the movement of the clumps. We estimated this threshold analytically and compare our findings to radiative transfer simulations with a range of covering factors, clump column densities, radii, and motions. Our results suggest that (i) the success in fitting observed Ly $\alpha$ spectra using homogeneous "shell models" (and the corresponding failure of multiphase models) hints at the presence of very small-scale structure in neutral gas, which is in agreement within a number of other observations; and (ii) the recurrent problems of reproducing realistic line profiles from hydrodynamical simulations may be due to their inability to resolve small-scale structure, which causes simulations to underestimate the effective covering factor of neutral gas clouds.
\end{abstract}

Key words. radiative transfer - ISM: clouds - galaxies: ISM - line: formation - scattering - galaxies: high-redshift

\section{Introduction}

Several observations highlight the presence of tiny, unresolved structure in atomic gas across a wide range of astrophysical environments. For instance, the wide, smooth emission lines in quasar spectra suggest the atomic gas close to the black hole has both a suprathermal velocity dispersion but low volume filling factor (e.g., Rees 1987; Arav et al. 1997). Moreover, studies of the diffuse gas in the halos of massive galaxies at redshifts $z \sim 2-3$ routinely find that these galaxies are filled with tiny clouds of neutral gas, again with a high covering factor, but with a low overall volume filling factor (e.g., Rauch et al. 1999; Cantalupo et al. 2014; Hennawi et al. 2015; Cai et al. 2017). Similar evidence for tiny-scale structure in neutral gas may be found in galactic winds and in high-velocity clouds in the Milky Way (see, e.g., McCourt et al. 2016, for a summary).

The physical origin of these clumps has been investigated recently by McCourt et al. (2016), who have found that cooling gas clouds are prone to rapid fragmentation akin to the Jeans instability. These authors have suggested this fragmentation rapidly "shatters" cold gas into tiny cloudlets of a characteristic size $l \sim 0.1 \mathrm{pc}\left(n / \mathrm{cm}^{-3}\right)^{-1}$, or equivalently a column density $N_{\text {cloudlet }} \sim 10^{17} \mathrm{~cm}^{-2}$. McCourt et al. (2016) have argued that this length scale is consistent with a number of observational upper limits, but unfortunately such small scales are extremely difficult to probe directly in distant objects. In this paper, we show

\footnotetext{
$\star$ The movie associated to Fig. B.2 is available at http://www . aanda.org

$\star \star$ Hubble fellow.
}

that radiative transfer of the resonant $\operatorname{Ly} \alpha$ line can indeed probe sub-parsec scales, even in distant galaxies.

There are several reasons why the Ly $\alpha$ emission line hydrogen is an ideal probe for tiny-scale structures. As the most prominent transition line of the most abundant element, Ly $\alpha$ is a sensitive probe of neutral gas enabling us to study even the most distant objects in the Universe. Recently, instruments such as MUSE (Bacon et al. 2010) reveal the ubiquity of Ly $\alpha$ emission throughout the observable space. In particular, Ly $\alpha$ is used to study our galactic neighborhood (Hayes 2015), galaxies at the peak of cosmic star formation (Barnes et al. 2014), and the later stages of reionization (Dijkstra 2014).

Apart from this practical reason, the resonant nature of the Ly $\alpha$ transition gives Ly $\alpha$ observations a potentially great constraining power in studying otherwise unresolvable structure. This is due to the strong frequency dependence of the neutral hydrogen scattering cross section, which leads to many orders of magnitude of variation in the photon mean free path. For instance, in a medium with one neutral hydrogen atom per $\mathrm{cm}^{-3}$, a Ly $\alpha$ photon travels on average only $\sim 1 \mathrm{AU}$ if it is in the core of the line; however, this distance grows by nearly five orders of magnitude to $\sim 0.5 \mathrm{pc}$ if the frequency is shifted merely five Doppler widths $\left(\sim 60 \mathrm{~km} \mathrm{~s}^{-1}\right)$ away from line center. The mean free path is therefore also sensitive to gas motions on the scale of $\sim(1-100) \mathrm{km} \mathrm{s}^{-1}$, providing powerful constraints on the kinematic properties of galaxies and their surrounding environments.

In this paper, we revisit Ly $\alpha$ radiative transfer through a simplified clumpy medium consisting of spherical "clouds" of neutral hydrogen embedded in an ionized surrounding medium. While this setup has been considered many times before 


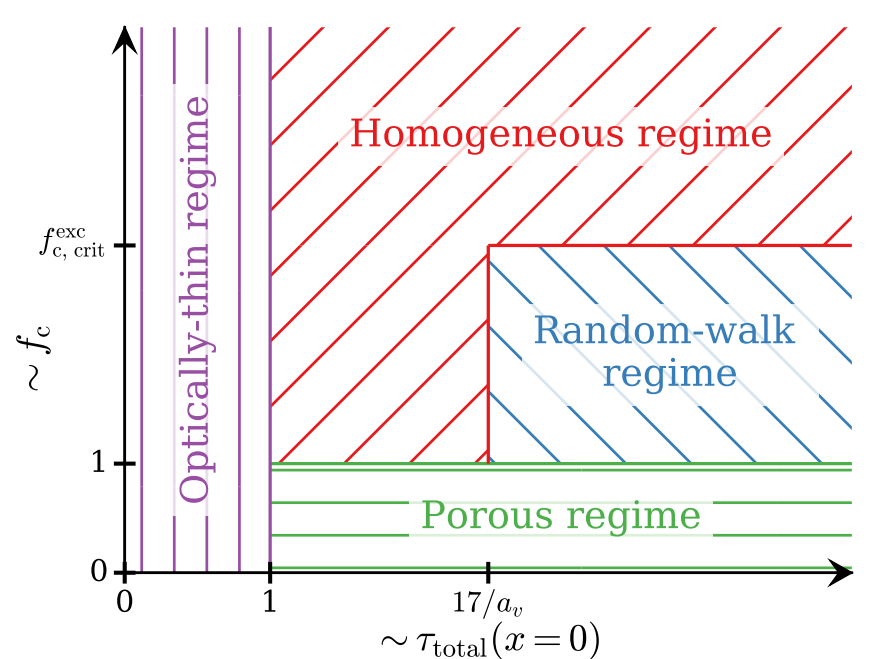

Fig. 1. Sketch of radiative transfer regimes in a static, clumpy medium discussed in Sect. 2.3. The $x$-axis shows the total optical depth at line center and $y$-axis the covering factor $f_{\mathrm{c}}$.

(e.g., Neufeld 1991; Hansen \& Oh 2006; Laursen et al. 2013; Duval et al. 2014; Gronke \& Dijkstra 2016), all of these previous studies have focused on a part of the parameter space with a relatively low $(\lesssim 10)$ number of clumps per sightline. In light of the observations and shattering scenario discussed above, we now consider the limit with many more clouds per sightline, exploring the full range from $\sim 1$ to $\sim 1000 \mathrm{~s}$. We show that this has tremendous influence on the propagation of $\operatorname{Ly} \alpha$ and we provide simple scaling relations that enable simple, order-of-magnitude calculations for Ly $\alpha$ radiative transfer through clumpy media.

Our paper is structured as follows. In Sect. 2, we discuss the problem analytically and estimate the expected results. In Sect. 3 we describe briefly our Ly $\alpha$ radiative transfer calculations and introduce our model. We present the simulation results in Sect. 4 with particular focus on the spectral shape and the Ly $\alpha$ escape fraction as well as their connections to a corresponding homogeneous medium. We then discuss the results in Sect. 5 before we conclude in Sect. 6 .

\section{Analytic results}

We find several distinct regimes for $\operatorname{Ly} \alpha$ radiative transfer in multiphase media, which we summarize in Fig. 1. In this section, we describe the physics relevant to each regime and provide analytic estimates for the boundaries separating them. Since it proves essential for our analysis, we first review some general results about Ly $\alpha$ escape from a homogeneous slab (Sect. 2.2) before describing radiative transfer in clumpy medium (Sect. 2.3). We confirm these analytic results numerically in Sect. 4 using Monte Carlo radiative transfer simulations.

\subsection{Definitions and notation conventions}

The basics of Ly $\alpha$ radiative transfer have been described in the literature (e.g., recently in a review by Dijkstra 2014) and are not repeated in detail here. Instead, we summarize the most relevant quantities for our present applications.

- We express the photon frequency $v$ in terms of its Doppler parameter

$x=\frac{v-v_{0}}{\Delta v}$, where $v_{0} \approx 2.47 \times 10^{15} \mathrm{~s}^{-1}$ is the frequency at line center, and $\Delta v_{\mathrm{D}}=v_{\text {th }} v_{0} / c=\sqrt{2 k_{\mathrm{B}} T / m_{\mathrm{H}}} v_{0} / c$ is the line width due to thermal motions of the atoms.

- Temperature dependence is expressed through the Voigt parameter

$a_{v}=\frac{\Delta v_{\mathrm{L}}}{2 \Delta_{\mathrm{D}}} \approx 4.7 \times 10^{-4}\left(\frac{T}{10^{4} \mathrm{~K}}\right)^{-1 / 2}$.

Here, $\Delta v_{\mathrm{L}}=9.939 \times 10^{7} \mathrm{~s}^{-1}$ is the natural (i.e., quantum mechanical) line broadening due to the finite lifetime of the transition.

- The Ly $\alpha$ cross section of neutral hydrogen is

$$
\begin{aligned}
\sigma_{\mathrm{HI}}(x, T) & =\sigma_{0} H\left(a_{v}, x\right) \\
& =\frac{\sigma_{0} a_{v}}{\pi} \int_{-\infty}^{\infty} \mathrm{d} y \frac{\mathrm{e}^{-y^{2}}}{(y-x)^{2}+a_{v}^{2}},
\end{aligned}
$$

where $\sigma_{0} \approx 5.895 \times 10^{-14}\left(T / 10^{4} \mathrm{~K}\right)^{-1 / 2} \mathrm{~cm}^{2}$ denotes its value at line center and $H\left(a_{v}, x\right)$ is the Voigt function, which can be approximated as $H\left(a_{v}, x\right) \sim \mathrm{e}^{-x^{2}}$ in the core of the line and as $\sim a_{v} /\left(\sqrt{\pi} x^{2}\right)$ in the wing of the line. The transition occurs at a frequency $x_{*} \approx 3.26$ for $T=10^{4} \mathrm{~K}$. The normalized Voigt distribution $\phi(x)=H\left(a_{v}, x\right) / \sqrt{\pi}$ represents the probability of a photon in the frequency interval $[x \pm \mathrm{d} x / 2]$ to interact with an atom.

- The optical depth per length $d$ is, hence,

$\tau(x)=\int_{0}^{d} \mathrm{~d} s \sigma_{\mathrm{HI}}(x) n_{\mathrm{HI}}(s)$,

where $n_{\mathrm{HI}}$ denotes the number density of neutral hydrogen atoms. We did not include the contribution of dust in the above expression as its impact is modeled in the post processing (see Sect. 4.5 for details).

\subsection{Radiative transfer in a homogeneous slab}

Since it is crucial for our analytic work below, we briefly review some classical solutions for $\operatorname{Ly} \alpha$ radiative transfer in a semi-infinite (that is, only one dimension is finite) slab with halfheight $B$ and optical depth $\tau_{0}$.

Ly $\alpha$ escape can be seen as a random walk in both real space and frequency space, as every scattering event (that is, absorption and quick re-emission from a neutral hydrogen atom) alters the frequency and direction of the $\operatorname{Ly} \alpha$ photon. However, because of the large value of $\sigma_{0}$, the mean free path of a photon close to line center is very small $\left(\lambda_{\mathrm{mfp}} \sim 5.5 \times 10^{-6}\left(\mathrm{n} / \mathrm{cm}^{-3}\right)^{-1} \mathrm{pc}\right.$ for $\left.T=10^{4} \mathrm{~K}\right)$, and most scatterings are spatially close to each other. Thus, the vast majority of scatterings do not contribute significantly toward the escape of the Ly $\alpha$ photon (at least in optically thick media ${ }^{1}$ ). Instead, Adams (1972) found that Ly $\alpha$ photons escape in several consecutive wing scatterings where the mean free path is significantly enhanced (for instance, $\lambda_{\mathrm{mfp}} \sim 0.48 \mathrm{pc}$ at $x=5$ for the above setup). The random walk in

1 For lower optical depths (when $\tau\left(x_{*}\right) \lesssim 1$ ), an escape in single flight is possible even in the Doppler core. Such escape occurs via rare scattering events when a photon near line center encounters a fast moving atom with large velocities perpendicular to the direction of the photon. When this photon is re-emitted, it is far from line center, and if $\tau(x) \approx \tau_{0} \mathrm{e}^{-x^{2}}<1$, it can escape (also see Sect. 2.3.3). 
frequency space is therefore crucial to the escape of $\operatorname{Ly} \alpha$. These series of wing scatterings are referred to as excursion and this is thought of as the common way $\operatorname{Ly} \alpha$ photons propagate in an astrophysical context.

To estimate the average displacement per "excursion", one has to take into account its random walk in frequency. Specifically, a Ly $\alpha$ photon in the wing of the line at frequency $x$ has a slight tendency to return to the core of the line with mean frequency shift per scattering event of $-1 / x$ (Osterbrock 1962). This means it scatters $\sim x^{2}$ times before returning to the core with a mean free path of $l=B \sigma_{0} /\left(\sigma_{\mathrm{HI}}(x) \tau_{0}\right)=B /\left(H(x) \tau_{0}\right)$ between each scatter. Since an excursion itself can be seen as a random walk, Adams (1972) obtained $d_{\mathrm{exc}}=\sqrt{N_{\mathrm{sct}, \mathrm{exc}} l}=x B /\left(H(x) \tau_{0}\right)$ as mean distance per excursion. Furthermore, by using the wing approximation $H(x) \sim a_{v} /\left(\sqrt{\pi} x^{2}\right)$ described above, and setting $d_{\text {exc }}=\sqrt{3} B$ (where the factor $\sqrt{3}$ arises due to geometrical considerations; Adams 1975), he obtained

$x_{\mathrm{esc}}=\left(\tau_{0} a_{v} \sqrt{3 / \pi}\right)^{1 / 3} \approx 6.5\left(\frac{N_{\mathrm{HI}}}{10^{19} \mathrm{~cm}^{-2}} \frac{10^{4} \mathrm{~K}}{T}\right)^{1 / 3}$

as an expression for the most likely escape frequency.

Adams (1972) continued to calculate the number of scatterings it takes for a photon to reach a frequency $|x| \geq x_{\text {esc }}$, which allows for escape. In an optically thick medium, photons undergo many scatterings and the frequency distribution $J(x)$ is roughly constant ${ }^{2}$. Thus, the probability to find an arbitrary photon with frequency in the interval $[x \pm \mathrm{d} x / 2]$ is $\sim \phi(x) \mathrm{d} x$ (complete redistribution approximation ${ }^{3}$ ). However, a given photon scatters $\sim x^{2}$ times at the frequency $\sim x$. Consequently, $\sim x^{2}$ scattering events are not into a frequency interval, which allows for escape, and thus the probability of scattering into $[x \pm \mathrm{d} x / 2]$ is $\sim \phi(x) / x^{2} \mathrm{~d} x$. This implies a cumulative escape probability

$P_{\mathrm{esc}}=2 \int_{x_{\mathrm{esc}}}^{\infty} \mathrm{d} x \frac{\phi(x)}{x^{2}}=\frac{2 a_{v}}{3 \pi x_{\mathrm{esc}}^{3}}$

where in the last equality we used the wing approximation for $\phi(x)$. The number of scatterings required to escape is $1 / P_{\mathrm{esc}}$ and plugging in $x_{\text {esc }}$ from Eq. (5) one obtains

$N_{\mathrm{sct}}^{\mathrm{esc}} \approx 4.6 \tau_{0} \approx 2.7 \times 10^{6}\left(\frac{N_{\mathrm{HI}}}{10^{19} \mathrm{~cm}^{-2}}\right)\left(\frac{T}{10^{4} \mathrm{~K}}\right)^{-1 / 2}$.

This relation differs only by a factor of a few with the exact solution of Harrington (1973) which has been backed up by numerical results (e.g., Bonilha et al. 1979; Dijkstra et al. 2006).

In summary, Adams (1972) found that a typical Ly $\alpha$ photon leaving an optically thick slab scatters a large number of times essentially in place (Eq. (7)), until it reaches the frequency $x_{\text {esc }}$ (Eq. (5)), after which it escapes undergoing $N_{\text {sct }}^{\text {exc }} \sim x_{\text {esc }}^{2}$ scattering interactions in the wing of the line.

\footnotetext{
2 Like any diffusive process, frequency diffusion can be represented by a Fokker-Planck equation, for which the steady-state solution is $J(x)=$ const. This is independent of the form of the frequency diffusion coefficient (and thus independent of the redistribution function).

3 This approximation holds only for $|x| \lesssim x_{\text {esc }}$, beyond which photons leave the system and $J(x)$ tends toward zero (over the intervals $\pm\left[x_{\text {esc }}, 2 x_{\text {esc }}\right]$, Adams 1972). Taking this into account only changes the pre-factors by order unity.
}

\subsection{Radiative transfer in clumpy medium}

Resonant line transfer in a clumpy medium has fundamentally different behavior than in a homogeneous medium because much of the distance can be traversed in the optically thin medium between the clumps. As we discussed in the previous section, owing to its highly variable interaction cross section, Ly $\alpha$ escapes through "excursion" in regimes for which the mean free path at the initial frequency is short. In a multiphase medium, however, a significant fraction of the volume may have no neutral hydrogen at all. The gas opacity thus varies strongly with position, even at line center. This opens up an alternate escape route in which Ly $\alpha$ photons "solve the maze" by scattering into the optically thin medium between clouds. This possibility is essential to consider, since astrophysical systems such as the ISM and CGM are thought to have a multiphase nature (e.g., McKee \& Ostriker 1977).

\subsubsection{Model parameters}

In this section, we describe the expected propagation of Ly $\alpha$ photon in a clumpy medium, which we model using spherical clumps of radius $r_{\mathrm{cl}}$ and HI number density $n_{\mathrm{HI}, \mathrm{cl}}$ placed in an otherwise empty, semi-infinite slab of height $2 B$. In what follows, we use the clump column density $N_{\mathrm{HI}, \mathrm{cl}}=r_{\mathrm{cl}} n_{\mathrm{HI}, \mathrm{cl}}$ and optical depth $\tau_{\mathrm{cl}}(x, T)=N_{\mathrm{HI}, \mathrm{cl}} \sigma_{\mathrm{HI}}(x, T)$ as convenient notation. The most important parameter of a clumpy medium is the covering factor $f_{\mathrm{c}}$, which describes the average number of clumps per orthogonal sightline between the midplane and the surface of the slab. These sightlines intercept a column density of $N_{\mathrm{HI}, \text { total }}=\frac{4}{3} f_{\mathrm{c}} N_{\mathrm{HI}, \mathrm{cl}}$ where the factor $4 / 3$ is due to the spherical geometry of the clumps ${ }^{4}$.

\subsubsection{Escape regimes}

In a static, clumpy medium several regimes are possible for the escape of a Ly $\alpha$ photon. We introduced some of these regimes in Gronke et al. (2016), but further describe each regime below. Furthermore, we sketch (i) under which circumstances each regime is active; (ii) on average, how many clumps a photon encounters $N_{\mathrm{cl}}$; and (iii) which emergent spectrum can be expected. Additionally, Fig. 1 provides a visual overview of the regimes, and a similar overview for a non-static setup is given in Appendix A.

- Porous regime. If a substantial number of sightlines do not intercept any clumps, many photons will not scatter and simply escape at their intrinsic frequency. The fraction of sightlines without any clumps can be estimated assuming the clumps are Poisson distributed with mean $f_{\mathrm{c}}$ yielding $\exp \left(-f_{\mathrm{c}}\right)$ (cf. Gronke \& Dijkstra 2016; Dijkstra et al. 2016). This area of the parameter space has been explored in previous work (Hansen \& Oh 2006; Laursen et al. 2013; Gronke \& Dijkstra 2016) and is of interest as the empty sightlines allow for ionizing photon escape (Verhamme et al. 2015; Dijkstra et al. 2016) and might allow for directionally dependent photon escape (Gronke \& Dijkstra 2014). This is the regime suggested by cosmological simulations of the CGM (e.g., Faucher-Giguère et al. 2015; Liang et al. 2016), although that may be a consequence of their limited resolution, which strongly limits the number of clumps to be no more than $\sim$ a few.

4 The mean path length through a sphere of radius $r$ is volume/area $=$ $4 / 3 \pi r^{3} /\left(\pi r^{2}\right)=4 / 3 r$. 
Table 1. Summary of the regimes found in a static, clumpy medium.

\begin{tabular}{|c|c|c|c|c|}
\hline Name & Requirement $^{a}$ & $N_{\mathrm{cl}}^{b}$ & $x_{\mathrm{p}}{ }^{c}$ & Description \\
\hline Free-streaming & $\tau_{0, \text { total }} \lesssim 1$ & $\propto f_{\mathrm{c}}$ & $\sim 0$ & Photons stream through clumps \\
\hline Porous & $\left(\tau_{0, \text { total }} \gtrsim 1\right) \wedge\left(f_{\mathrm{c}} \lesssim 1\right)$ & $\sim 0$ & $\sim 0$ & Escape through holes without interaction \\
\hline Random-walk & $\left(\tau_{0, \text { total }} \gtrsim 17 / a_{v}\right) \wedge\left(1 \lesssim f_{\mathrm{c}} \lesssim f_{\mathrm{c}, \text { crit }}^{\mathrm{exc}}\right)$ & $\propto f_{\mathrm{c}}^{2}$ & $\sim 0$ & Scatter off clumps until escape \\
\hline \multirow[t]{2}{*}{ Homogeneous } & $\left(\tau_{0, \text { total }} \gtrsim 17 / a_{v}\right) \wedge\left(f_{\mathrm{c}} \gtrsim f_{\mathrm{c}, \text { crit }}^{\mathrm{exc}}\right)$ & $\propto f_{\mathrm{c}}$ & $\sim x_{\mathrm{esc}}{ }^{A}$ & Excursion-like escape \\
\hline & $\left(17 / a_{v} \gtrsim \tau_{0, \text { total }} \gtrsim 1\right) \wedge\left(f_{\mathrm{c}} \gtrsim f_{\mathrm{c}, \text { crit }}^{\mathrm{exc}}\right)$ & $\propto f_{\mathrm{c}}$ & $\sim x_{*}^{B}$ & Escape in a single flight \\
\hline
\end{tabular}

Notes. The regimes are described in detail in Sect. 2.3. ${ }^{(a)}$ Parameter space of regime. Visualized in Fig. 1. ${ }^{(b)}$ Average number of clumps a photon encounters before escape. ${ }^{(c)}$ Dominant frequency of escape. ${ }^{(A)}$ Escape frequency from a homogeneous slab (Eq. $\left.(5)\right)$. ${ }^{(B)}$ Boundary between core and wing of the line. $x_{*} \sim 3.26$ for $T=10^{4} \mathrm{~K}$.

- Random walk regime. If the clumps are optically thick to the photons, i.e., $\tau_{\mathrm{cl}} \gtrsim 1$, the photons are expected to scatter at every clump encounter. When $\tau_{\mathrm{cl}} \gg 1$ the photon scatters close to the surface of each clump and effectively random walks between the clumps, rather than through the clumps. This regime was studied by Neufeld (1991) and by Hansen \& Oh (2006). In this "random walk regime" the number of clumps a photon intercepts scales as $\propto f_{\mathrm{c}}^{2}$. This is because after $N_{\mathrm{cl}}$ interactions a photon has traveled on average a distance $\sqrt{N_{\mathrm{cl}}} B / f_{\mathrm{c}}$ away from the midplane. Thus, to escape this distance has to be $\sim B$, which yields $N_{\mathrm{cl}}=f_{\mathrm{c}}{ }^{2}$. Hansen \& Oh (2006) found the scaling to be

$N_{\mathrm{cl}} \sim f_{\mathrm{c}}^{2}+f_{\mathrm{c}}$

with pre-factors of order unity that depend somewhat on the geometry (see Hansen \& Oh 2006, for details).

- Optically thin regime. If the clumps are, on the other hand, optically thin to the intrinsic radiation $\left(\tau_{0, \mathrm{cl}} \lesssim 1\right)$, not every cloud interception necessarily causes the photon to scatter. In particular, the probability of a scattering event to happen in this case is $1-\exp \left(-\tau_{0, \mathrm{cl}}\right) \approx \tau_{0, \mathrm{cl}}$. This implies that to describe the expected scaling in this regime, we can replace in the above considerations $f_{\mathrm{c}}$ by $\tau_{0, \mathrm{cl}} f_{\mathrm{c}}$. As in this regime $\tau_{0, \mathrm{cl}} \lesssim 1$, this corresponds to decrease effectively the prefactors in Eq. (8). Specifically, if all clumps in a given sightline are optically thin, the intrinsic photons at line center, that is, $\tau_{0 \text {,total }} \equiv 4 / 3 f_{\mathrm{c}} \tau_{0, \mathrm{cl}} \lesssim 1$, most Ly $\alpha$ photons do not interact before escaping. This means they simply stream through all the clumps (leading to $N_{\mathrm{cl}} \propto f_{\mathrm{c}}$ ) keeping their intrinsic frequency (i.e., a peak frequency of $x_{\mathrm{p}} \sim 0$ ). We call this an optically thin regime.

- Homogeneous regime. Since $\tau_{\mathrm{cl}}$ depends strongly on the frequency of the photon, which changes throughout the scattering process, Ly $\alpha$ might also escape from clumpy media in a frequency excursion as discussed in the homogeneous slab. In particular, during the course of the $\sim f_{\mathrm{c}}{ }^{2}$ scatterings needed to random walk through the clumpy medium, the photon may scatter far enough into the wing of the line to escape the medium in a single excursion as described in Sect. 2.2. If this happens, most clumps become optically thin for the photon and one can generalize the argument made above when describing the optically thin regime by replacing $\tau_{0, \mathrm{cl}}$ by $\tau_{\mathrm{cl}}(x)$. Since this possibility becomes increasingly likely with every scattering event, we anticipate that above some critical value of $f_{\mathrm{c}}$, clumpy media behave like homogeneous slabs in the sense that photos escape via frequency excursion.

Conclusively, the four regimes are different in the photon's preferential escape route, which depends in the static case on the covering factor $f_{\mathrm{c}}$ and the optical depth of individual clumps. Each escape route implies that the photon experiences a clumpy medium differently, which leaves a clear imprint on the emergent spectrum. One way to characterize these regimes is the optically thin and porous regimes represent escape without significant interaction, while the random walk and homogeneous regimes represent escape primarily via spatial or frequency diffusion, respectively. Table 1 provides a brief summary of the different regimes.

\subsubsection{Division between the regimes}

In the last section we introduced the four different routes for a Ly $\alpha$ photon to escape from a clumpy medium. We also briefly discussed the physical conditions under which each escape route is favored. In this section, we quantify these boundaries more precisely.

We denote the boundary between the homogeneous and other regimes with $f_{\mathrm{c} \text {, crit }}$, and specifically to the random walk regime with $f_{\mathrm{c}, \mathrm{crit}}^{\text {exc }}$. Physically, this value characterizes the critical number of clumps per sightline when a excursion-like escape becomes faster than a random-walk diffusion. In order to find this boundary we follow this argument and compute the criteria for when it is possible for the photons to stream through the clumps ${ }^{5}$. As stated above, the characteristic escape frequency is given by Eq. (5), and in a clumpy medium the total line center optical depth is $\tau_{0}=4 / 3 f_{\mathrm{c}} N_{\mathrm{HI}, \mathrm{cl}} \sigma_{0}$. The transition occurs when photons can stream through individual clumps, that is when $4 / 3 \tau_{\mathrm{cl}}\left(x_{\mathrm{esc}}\right)=1$. Using the wing approximation for the Ly $\alpha$ cross section, this yields

$f_{\mathrm{c}, \mathrm{crit}}^{\mathrm{exc}}=\frac{2 \sqrt{a_{v} \tau_{0, \mathrm{cl}}}}{3 \pi^{1 / 4}} \approx\left(\frac{N_{\mathrm{HI}, \mathrm{cl}}}{10^{17} \mathrm{~cm}^{-2}}\right)^{1 / 2}\left(\frac{T}{10^{4} \mathrm{~K}}\right)^{-1}$.

For optically thinner medium, an escape through excursion is not possible as a frequency shift into the wings of the lines leads to immediate escape. Specifically, this happens if the wings become optically thin, i.e., if $\sqrt{3} \tau\left(x_{*}\right)<1$, which translates to

5 This derivation of $f_{\mathrm{c}, \text { crit }}$ in a static setup is complementary to that presented in Gronke et al. (2016), in which we used a timescale argument. 
$\sqrt{3} \tau_{0} a_{v}<\sqrt{\pi} x_{*}^{2} \approx 18.78$, where we included factors of $\sqrt{3}$ due to the rectangular geometry. This transition happens for an homogeneous medium as well as a clumpy medium and sets a lower limit to $f_{\mathrm{c} \text {, crit }}$. However, if the individual clumps possess an optical depth at line center of $\tau_{0, \mathrm{cl}} \lesssim 1$, not every clump encounter leads necessarily to a scattering, and thus introduces the additional factor of $1-\mathrm{e}^{-\tau_{0, \mathrm{cl}}}$ (as described for the optically thin regime in Sect. 2.3.3).

In conclusion, we expect the transition to the homogeneous regime to occur if

$f_{\mathrm{c}, \text { crit }}= \begin{cases}f_{\mathrm{c}, \text { crit }}^{\mathrm{exc}}=\frac{2 \sqrt{a_{v} \tau_{0, \mathrm{cl}}}}{3 \pi^{1 / 4}} & \text { for } \sqrt{3} a_{v} \tau_{0}>\sqrt{\pi} x_{*}^{2} \\ \frac{f_{\mathrm{c}, \text { crit }}^{\mathrm{sf}}}{1-\mathrm{e}^{-\tau_{0, \mathrm{cl}}}} & \text { otherwise, }\end{cases}$

where $f_{\mathrm{c} \text {, crit }}^{\mathrm{sf}}=2 x_{*} / 3^{5 / 4} \approx 1.65$ is due to continuity of $f_{\mathrm{c} \text {, crit }}$ at $\sqrt{3} a_{v} \tau_{0}=\sqrt{\pi} x_{*}^{2}$, that is, at the transition from excursion to single-flight escape.

\subsubsection{Non-static case}

Since the Ly $\alpha$ cross section depends sensitively on the frequency $x$, clump motions can dramatically influence radiative transfer. If a clump moves with a velocity $\gtrsim x_{*} v_{\text {th }} \sim 50 \mathrm{~km} \mathrm{~s}^{-1}$, a single clump interaction puts the photon far enough into the wing of the line to allow the photon to escape directly in a single excursion. This possibility is important to consider because random velocities $v \sim 100 \mathrm{~km} \mathrm{~s}^{-1}$ may be typical for the CGM in galaxies and in galactic winds, and velocities $v \sim 1000 \mathrm{~km} \mathrm{~s}^{-1}$ may be typical in the regions around black holes. This means that for large $f_{\mathrm{c}}$ the medium behaves as a slab with an increased temperature of

$T_{\text {eff }}=T+\frac{\sigma_{\mathrm{cl}}^{2} m_{\mathrm{H}}}{2 k_{\mathrm{B}}}$,

where $\sigma_{\mathrm{cl}}$ is the $1 \mathrm{D}$ velocity dispersion of the clumps.

For a lower number of clumps the overall velocity distribution is not well sampled, which leads to sightlines with no clumps in the core of the line. In this case the photons escape without any clump interaction. We can estimate this to happen if the mean separation of two clumps in velocity space becomes larger than the velocity range over which a clump can provide $\tau_{\mathrm{cl}} \gtrsim 1$. For a Gaussian velocity distribution with variance of $\sigma_{\mathrm{cl}}^{2}$, the average separation is approximately given by $\sigma_{\mathrm{cl}} /\left(\alpha f_{\mathrm{c}}\right)$ where $\alpha$ is the fraction of clumps within core of the velocity distribution, i.e., in our case $\alpha \approx 0.68$. Consequently, the transition to the homogeneous regime for randomly moving clumps occurs at $4 / 3 \tau_{\mathrm{cl}}\left(\sigma_{\mathrm{cl}} /\left(\alpha f_{\mathrm{c}} v_{\mathrm{th}}\right)\right)=1$, which - using the core approximation and including geometrical factors - can be written as a critical covering factor for the randomly moving case

$f_{\mathrm{c}, \text { crit }}= \begin{cases}\frac{\sigma_{\mathrm{cl}}}{\alpha v_{\mathrm{th}} \sqrt{\ln \left(4 / 3 \tau_{0, \mathrm{cl}}\right)}} & \text { if } f_{\mathrm{c}}>1 / \alpha \\ \frac{1}{\alpha} & \text { otherwise. }\end{cases}$

Here, the lower boundary of $1 / \alpha$ results simply from the requirement that at least one clump within the core of the velocity distribution function is necessary to sample the core of the velocity distribution. We expect, for larger covering factors, the system to behave as a homogeneous slab of temperature $T_{\text {eff }}$. See also Appendix A for more details about the expected behavior in the case of uncorrelated clump motion.
In the case of clumps with a systematic velocity structure (for instance, outflowing clumps), the above requirement of a well-sampled velocity field is fulfilled if the adjacent clump is optically thick to the Ly $\alpha$ photon, i.e., if $4 / 3 \tau_{\mathrm{cl}}\left(x_{\text {next }}\right) \gtrsim 1$, where $x_{\text {next }}$ depends on the exact velocity profile. For a linearly scaled (Hubble-like) outflow from 0 at midplane to $\left|v_{\max }\right|$ at the boundaries of the slab, we have $x_{\text {next }}=v_{\max } /\left(f_{\mathrm{c}} v_{\mathrm{th}}\right)$. In addition, a photon might be artificially forced into the wing of the line if $x_{\text {next }}>x_{*}$ owing to the sampling of the velocity field. This does not occur in a homogeneous medium, and thus, for a Hubble-like outflow the criterion to be fulfilled for the homogeneous regime is

$f_{\mathrm{c}, \text { crit }}= \begin{cases}\frac{\sqrt{\pi} v_{\max }^{2}}{a_{v} N_{\mathrm{HI}, \mathrm{total}} v_{\mathrm{th}}^{2} \sigma_{0}} & \text { if } v_{\max }>\hat{v}_{\text {max }} \\ x_{*} v_{\mathrm{th}} / v_{\max } & \text { otherwise, }\end{cases}$

where $\hat{v}_{\max }=v_{\text {th }}\left(a_{v} N_{\mathrm{HI}, \text { total }} x_{*} \sigma_{0} / \sqrt{\pi}\right)^{1 / 3}$.

\section{Numerical method}

\subsection{Ly $\alpha$ radiative transfer}

Because of the complexity of the resonant line transfer, Monte Carlo radiative transfer codes are commonly in use (e.g., Auer 1968; Ahn et al. 2002; Zheng \& Miralda-Escudé 2002). This algorithm works by following individual photon packages in a stochastic manner through real and frequency space until their escape. In this work, we used the code tlac, which has been used and described previously, for example, in Gronke \& Dijkstra (2014). In particular, we made use of tlac's features (i) to handle embedded spherical grids within a Cartesian grid; and (ii) employ a dynamical core-skipping scheme (as described in Smith et al. 2015; Gronke \& Dijkstra 2016). We also turned off the dynamical core skipping for a few models and checked that the emergent spectra are identical. We ran most setups using $\sim 10^{4}$ photon packages but occasionally used more to obtain a higher resolution spectrum.

\subsection{Model parameters}

Analogous to Sect. 2.3, our setup consisted of a slab with half-height $B$ in which we distributed non-overlapping spherical clumps with radius $r_{\mathrm{cl}}$ randomly in the box until a fraction of the total volume $F_{V}$ was filled ${ }^{6}$. This means the number density of clumps is $n_{\mathrm{cl}}=F_{V} /\left(4 / 3 \pi r_{\mathrm{cl}}^{3}\right)$, where $r_{\mathrm{cl}}$ is the clump radius. The connection between the volume filling factor $F_{V}$ and the previously introduced covering factor $f_{\mathrm{c}}$, which describes the average number of clumps a line orthogonal to the slab intercepts between the midplane and boundary of the box, is given by the integration along the finite axis of the slab, that is,

$f_{\mathrm{c}}=\int_{0}^{B} \mathrm{~d} r \pi n_{\mathrm{cl}} r_{\mathrm{cl}}^{2}=\frac{3 F_{V} B}{4 r_{\mathrm{cl}}}$.

The clumps are filled with neutral hydrogen with a number density of $n_{\mathrm{HI}, \mathrm{cl}}$ and temperature $T$, leading to a column density

\footnotetext{
6 Hansen \& Oh (2006) compared spherical clumps to random surface distributions and found their results to be only mildly affected by the shape of the clumps. In fact, they found the pre-factors of $N_{\mathrm{cl}}$ (Eq. (8)) to be sensitive to the clump geometry by factors of order unity but their results, if parametrized via $N_{\mathrm{cl}}$, were unchanged.
} 
Table 2. Overview of the model parameters.

\begin{tabular}{lll}
\hline \hline Symbol & Description & Considered values \\
\hline$B$ & Half-height of slab & $50 \mathrm{pc}$ \\
$r_{\mathrm{cl}}$ & Clump radius & $\left\{10^{-3}, 10^{-2}\right\} \mathrm{pc}$ \\
$f_{\mathrm{c}}{ }^{a}$ & Covering factor & {$[0,2000]$} \\
$T$ & Temperature & $10^{4} \mathrm{~K}$ \\
$N_{\mathrm{HI}, \mathrm{cl}}{ }^{b}$ & Clump HI column density & {$\left[10^{12}, 10^{22}\right] \mathrm{cm}^{-2}$} \\
$\sigma_{\mathrm{cl}}$ & $\begin{array}{l}\text { Clumps' velocities standard } \\
\text { deviation }\end{array}$ & {$[0,500] \mathrm{km} \mathrm{s}^{-1}$} \\
$\tau_{\mathrm{d,cl}}{ }^{b}$ & Clump (absorbing) dust & {$\left[10^{-4}, 1\right]$} \\
& optical depth & {$[0,5000] \mathrm{km} \mathrm{s}^{-1}$} \\
$v_{\mathrm{max}}$ & $\begin{array}{l}\text { Maximum clump outflow } \\
\text { velocity }\end{array}$ & \\
\cline { 2 - 3 }$F_{V}$ & Volume filling factor & {$[0,0.6]$} \\
$N_{\mathrm{HI}, \text { total }}{ }^{a}$ & HI column density of slab & {$\left[10^{11}, 10^{26}\right] \mathrm{cm}^{-2}$} \\
$n_{\mathrm{HI}, \mathrm{cl}}$ & $\begin{array}{l}\text { HI number density in } \\
\text { clumps }\end{array}$ & {$\left[10^{-5}, 10^{6}\right] \mathrm{cm}^{-3}$} \\
$\tau_{0, \mathrm{cl}}{ }^{b}$ & $\begin{array}{l}\text { Line center optical depth of } \\
\text { clump }\end{array}$ & {$\left[0.05,10^{9}\right]$} \\
$\tau_{0, \text { total }}{ }^{a}$ & $\begin{array}{l}\text { Total line center optical } \\
\text { depth }\end{array}$ & {$\left[10^{-3}, 10^{13}\right]$} \\
$\tau_{\mathrm{d}, \text { total }}{ }^{a}$ & $\begin{array}{l}\text { Total (absorbing) dust } \\
\text { optical depth }\end{array}$ & {$\left[0,10^{3}\right]$} \\
\hline
\end{tabular}

Notes. Above and below the horizontal line are the free and dependent parameters, respectively. ${ }^{(a)}$ From emitting plane to boundary of the slab, i.e., per $B .{ }^{(b)}$ From center to boundary of a clump, i.e., per $r_{\mathrm{cl}}$.

between the center of the clumps to their outskirts of $N_{\mathrm{HI}, \mathrm{cl}}=$ $r_{\mathrm{cl}} n_{\mathrm{HI}, \mathrm{cl}}$. As described in Sect. 2.3, this means on average the shortest path between the midplane and the boundary of the box intercepts a column density of

$N_{\mathrm{HI}, \text { total }}=\frac{4}{3} f_{\mathrm{c}} N_{\mathrm{HI}, \mathrm{cl}}=F_{V} B n_{\mathrm{HI}, \mathrm{cl}}$.

In general, we considered three cases: the static case with no motion, the randomly moving case, and an outflowing case. Astrophysical sources commonly show signs of turbulent motion (probed, e.g., through $\mathrm{H} \alpha$ line profiles; Herenz et al. 2016). For instance, turbulence in the ISM is thought to be driven by supernova explosions. Furthermore, outflows such as galactic winds are driven by star formation or AGN mediated feedback (probed, for instance, via absorption lines; Steidel et al. 2010; Rivera-Thorsen et al. 2015). The static case, on the other hand, allowed us to study the pure radiative transfer without the additional complications of Doppler shifts due to bulk motions.

In the randomly moving case, we assigned each clump a random velocity by drawing each component from a Gaussian with standard deviation $\sigma_{\mathrm{cl}}$. This represents a "white noise" spectrum with velocity differences that are statistically equally probable on all spatial scales. For the outflow, we chose a simple linear velocity scaling from $0 \mathrm{~km} \mathrm{~s}^{-1}$ to $v_{\max }$ at the midplane and boundary of the slab, respectively. We will investigate models with correlated turbulence and different velocity profiles in future work.

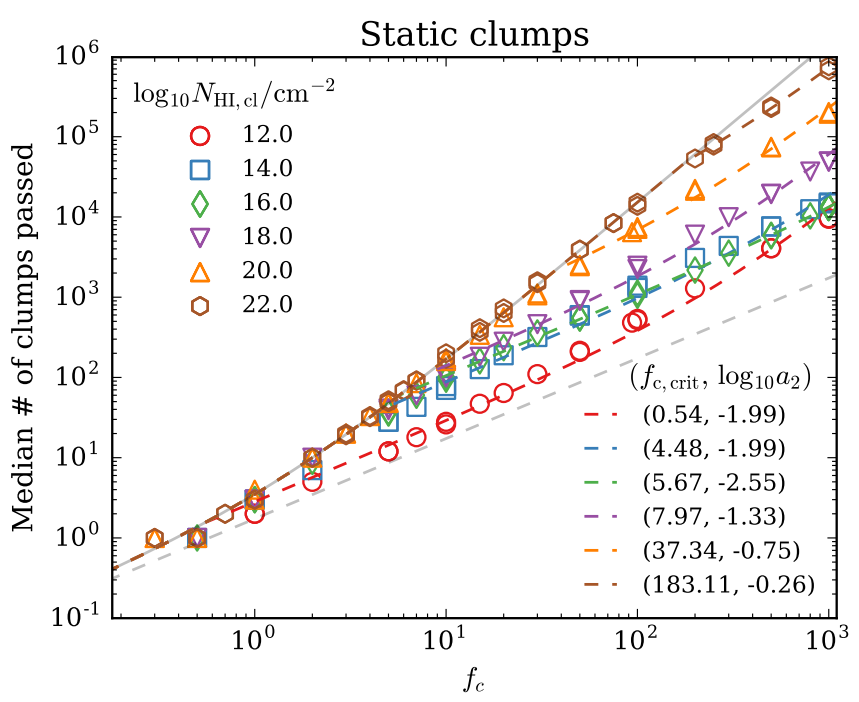

Fig. 2. Number of clumps passed vs. covering factor $f_{\mathrm{c}}$ for different clump column densities. The dashed lines show fits of Eq. (16) to the data points and the gray solid (dashed) line shows the limit with $f_{\text {c,crit }}>$ $10^{3}\left[N_{\mathrm{HI}, \mathrm{cl}} \rightarrow 0\right]$.

Furthermore, we studied two different emission sites for the Ly $\alpha$ photons: first, simply the midplane of the box and, secondly, randomly chosen emission within the clumps. While the former is useful to study merely the radiative transfer processes through the clumpy medium from an external source such as a star-forming region, the latter case represents a physically motivated scenario in which $\operatorname{Ly} \alpha$ are produced via recombination events within the clumps. Both scenarios might be responsible, for example, for the $\operatorname{Ly} \alpha$ halos found around galaxies (e.g., Dijkstra \& Kramer 2012; Mas-Ribas \& Dijkstra 2016).

\section{Numerical results}

In this section we present the results from our numerical radiative transfer simulations. In particular, we focus on three quantities, namely the number of clumps encountered by the photons $N_{\mathrm{cl}}$, and the emergent Ly $\alpha$ spectra. The value $N_{\mathrm{cl}}$ is a useful diagnostic, since we expect $N_{\mathrm{cl}} \sim f_{\mathrm{c}}^{2}$ for escape via random walk in position space, and $N_{\mathrm{cl}} \sim f_{\mathrm{c}}$ for escape via excursion and single flight as described in the previous section with the transition occurring at $f_{\mathrm{c}} \sim f_{\mathrm{c} \text {, crit }}$.

The section is approximately ordered by ascending complexity. In Sect. 4.1, we discuss the static case, in Sects. 4.2 and 4.3 we introduce random clump motions and outflows, respectively. Moreover, in Sect. 4.4 we change the emission site of the photons to be inside the clumps, which resembles a case of fluorescent emission. Finally, we study the effect of dust inside the clumps on the Ly $\alpha$ escape in a clumpy medium (Sect. 4.5), which we quantify through the Ly $\alpha$ escape fraction.

\subsection{Static case}

Figure 2 shows the number of clumps a Ly $\alpha$ photon passed through before escaping the box versus the covering factor $f_{\mathrm{c}}$, which we vary over $\sim 3$ orders of magnitude. Each symbol and color represents different values of $N_{\mathrm{HI}, \mathrm{cl}}$ and, thus, different clump optical depths at line center $\tau_{c l, 0}$ which we vary from $\sim 0.06$ (optically thin) to $\sim 6 \times 10^{8}$ (optically thick). We also ran each combination of $\left(N_{\mathrm{HI}, \mathrm{cl}}, f_{\mathrm{c}}\right)$ with two different cloud radii 


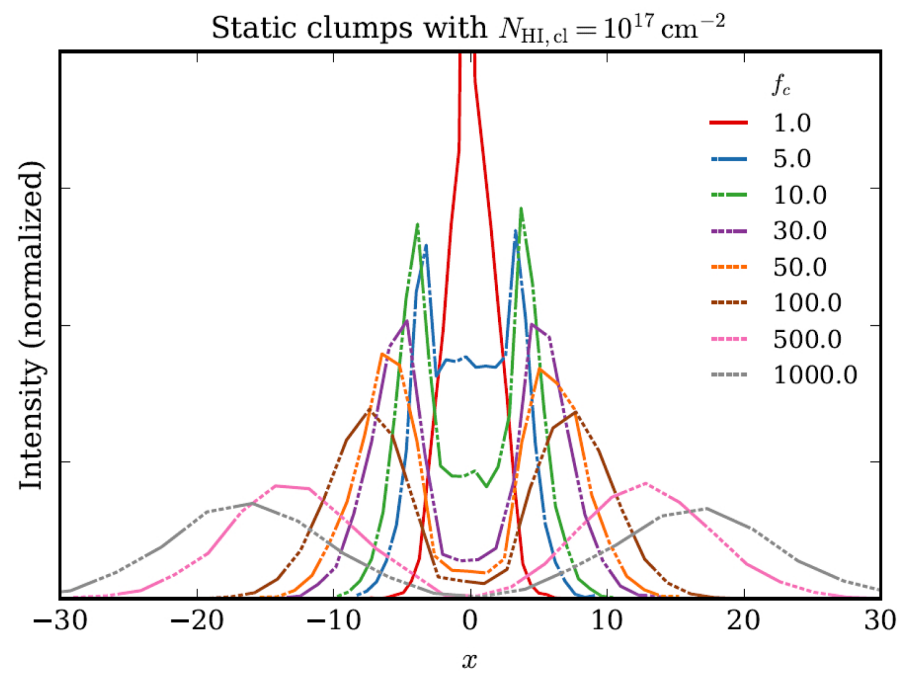

Fig. 3. Ly $\alpha$ spectra for a constant clump column density $N_{\mathrm{HI}, \mathrm{cl}}=$ $10^{17} \mathrm{~cm}^{-2}$ and various values of $f_{\mathrm{c}}$ (increasing $f_{\mathrm{c}}$ corresponds to an increased spectral width).

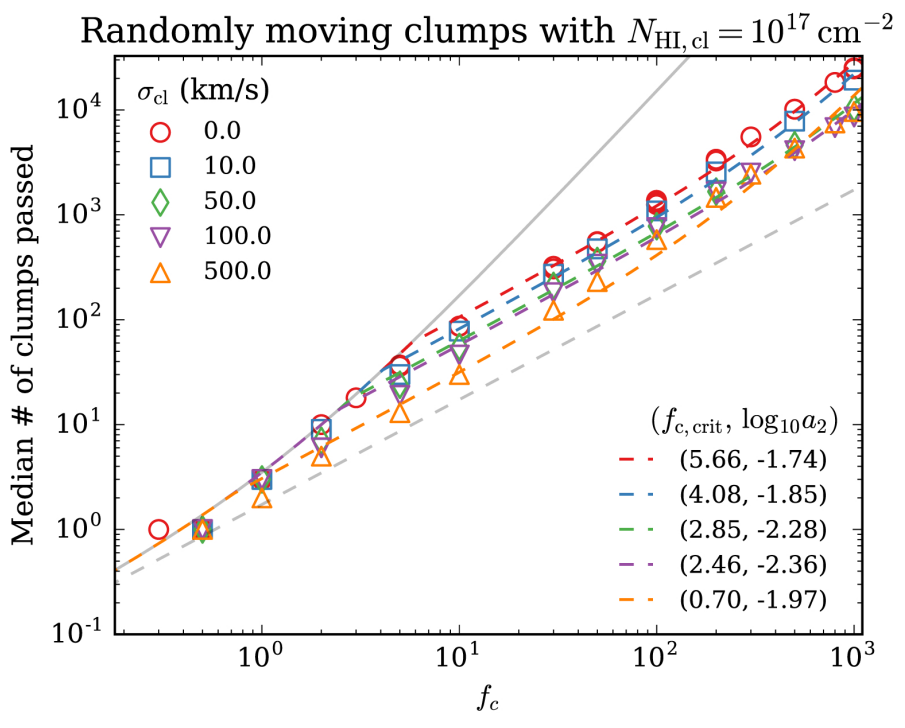

Fig. 4. Number of clumps passed vs. $f_{\mathrm{c}}$ for clumps with $N_{\mathrm{HI}, \mathrm{cl}}=$ $10^{17} \mathrm{~cm}^{-2}$ and uncorrelated, random motion with various $\sigma_{\mathrm{cl}}$. The dashed lines show fits of Eq. (16) to the data points and the gray solid line shows the limit with $f_{\mathrm{c}, \text { crit }}>10^{3}$.

$r_{\mathrm{cl}}=\left\{10^{-2}, 10^{-3}\right\} \mathrm{pc}$ to confirm that this parameter is not important (Hansen \& Oh 2006).

The dashed lines in the corresponding color show curves following

$N_{\mathrm{cl}}= \begin{cases}a_{1} f_{\mathrm{c}}^{2}+b_{1} f_{\mathrm{c}} & \text { for } f_{\mathrm{c}}<f_{\mathrm{c}, \text { crit }} \\ a_{2} f_{\mathrm{c}}^{2}+b_{2} f_{\mathrm{c}} & \text { for } f_{\mathrm{c}} \geq f_{\mathrm{c}, \text { crit }}\end{cases}$

with the resulting best-fit parameters for $\left(f_{\mathrm{c}, \text { crit }}, a_{2}\right)$ shown in the legend in each figure. Prior we fit the data points for $N_{\mathrm{HI}, \mathrm{cl}}=$ $10^{22} \mathrm{~cm}^{-2}$ for $f_{\mathrm{c}} \leq 100$ to determine $\left(a_{1}, b_{1}\right)=(3 / 2,2)$; the best-fit values are $(1.51,1.90)$ which - given the uncertainty - we rounded to the nearest convenient fraction for simplicity. These coefficients represent geometrical factors in the surface scattering regime, where clouds are optically thick, and thus independent of $N_{\mathrm{HI}, \mathrm{cl}}$. We directly verified this numerically. These values are then fixed for all $N_{\mathrm{HI}, \mathrm{cl}}$ in order to fit each $N_{\mathrm{HI}, \mathrm{cl}}$-curve for $\left(f_{\mathrm{c}, \text { crit }}, a_{2}\right)$, while $b_{2}$ is fixed by requiring continuity at $f_{\mathrm{c} \text {, crit }}$.
Figure 2 shows the resulting fits and the obtained values for $\left(f_{\mathrm{c}, \text { crit }}, a_{2}\right)$. The break in the scaling relation at $f_{\mathrm{c} \text {, crit }}$ is clearly visible; for visual aid, Fig. 2 also shows the $f_{\mathrm{c}}=a_{1} f_{\mathrm{c}}{ }^{2}+b_{1} f_{\mathrm{c}}$ curve from which the scaling departures for $f_{\mathrm{c}}>f_{\mathrm{c} \text {, crit }}$.

We found that the obtained $f_{\mathrm{c} \text {, crit }}$ for high column densities $\left(N_{\mathrm{HI}, \mathrm{cl}} \gtrsim 10^{20} \mathrm{~cm}^{-2}\right)$ matches the prediction from Sect. 2.3.3 reasonably well; this breaks down for lower optical depths. Also, for $N_{\mathrm{HI}, \mathrm{cl}}=10^{12} \mathrm{~cm}^{-2}$, i.e., when the clumps are always optically thin for Ly $\alpha$ photons, we obtained $a_{2} \approx \tau_{0, c l}^{2} \approx 0.010$ as discussed in Sect. 2. With increasing $N_{\mathrm{HI}, \mathrm{cl}}$ we found a decreasing $a_{2}$ to match the data. Thus, we can identify the escape regimes (characterized by the number of clumps encountered) described in the analytic model in Sect. 2. In summary, Fig. 2 shows that for $f_{\mathrm{c}}<f_{\mathrm{c}, \text { crit }}, N_{\mathrm{cl}} \propto f_{\mathrm{c}}{ }^{2}$ (as expected for a random walk), while for $f_{\mathrm{c}}>f_{\mathrm{c}, \text { crit }}, N_{\mathrm{cl}} \propto f_{\mathrm{c}}$ (as expected for escape through excursion).

Figure 3 shows the corresponding spectra for $N_{\mathrm{HI}, \mathrm{cl}}=$ $10^{17} \mathrm{~cm}^{-2}$. For this column density, we found $f_{\mathrm{c} \text {, crit }} \sim 2$, which corresponds roughly to the boundary between single and doublepeaked spectra. In particular, we recovered the spectral shape of Hansen \& Oh (2006) for $f_{\mathrm{c}} \ll f_{\text {c, crit }}$ while obtaining wide, double-peaked spectra with zero flux at line center for $f_{\mathrm{c}} \gg$ $f_{\mathrm{c} \text {, crit }}$. This means that the escape regimes not only impact the paths of the photons but also modify the escape frequencies and, hence, leave a clear observational signature on the emergent spectra.

\subsection{Random motion}

Figure 4 shows the $N_{\mathrm{cl}}-f_{\mathrm{c}}$ scaling relation in the case of random clump motion for a fixed clump's column density of $N_{\mathrm{HI}, \mathrm{cl}}=10^{17} \mathrm{~cm}^{-2}$. As conjectured in Sect. 2.3.4, compared to the static case the photons spend less time until escape and thus the number of clumps passed is smaller. This is because in the case of fast moving clumps, the photons escape either through "holes" in velocity space (where, $N_{\mathrm{cl}} \propto f_{\mathrm{c}}$ ) or via single flight (in which case also $N_{\mathrm{cl}} \propto f_{\mathrm{c}}$ ). Departures from that are either due to convergence to the static case (for $\sigma_{\mathrm{cl}} \rightarrow 0$ ) or when escape via single flight involves multiple surface scatterings (for $\left.f_{\mathrm{c}} \ll f_{\text {c, crit }}\right)$ when the interaction with another clump is nonnegligible.

In Fig. 5 we show the emergent spectra from this setup. In particular, we focus on the case with $f_{\mathrm{c}} \approx 1000$ and $N_{\mathrm{HI}, \mathrm{cl}}=$ $10^{16} \mathrm{~cm}^{-2}$ and four different values of clump velocity dispersion $\sigma_{\mathrm{cl}}$. Also in Fig. 5 we overlay spectra from homogeneous slabs with an effective temperature $T_{\text {eff }}$ (see Eq. (11)) corresponding to the respective value of $\sigma_{\mathrm{cl}}$. Clearly, the spectra match very well and, specifically, the peak separation. However, with increasing $T_{\text {eff }}$ the matches become worse, which makes sense since the wider velocity space is more poorly sampled.

\subsection{Outflows}

Figure 6 shows the $N_{\mathrm{cl}}-f_{\mathrm{c}}$ relation in the presence of linearly scaled outflows with maximum velocity $v_{\max }$ (as described in Sect. 3.2). We can see that a flattening of the curve still exists, which we interpret again as the transition between the "random walk" and "homogeneous regime". As expected with increasing outflow speed this threshold decreases.

Figure 7 illustrates the change in spectral shape when introducing outflows. In each subpanel, the solid lines show the emergent spectrum from the clumpy model and the dashed lines in matching color show those from a homogeneously filled slab 
Randomly moving clumps with $f_{c} \approx 1000, N_{\mathrm{HI}, \mathrm{cl}}=10^{16} \mathrm{~cm}^{-2}$

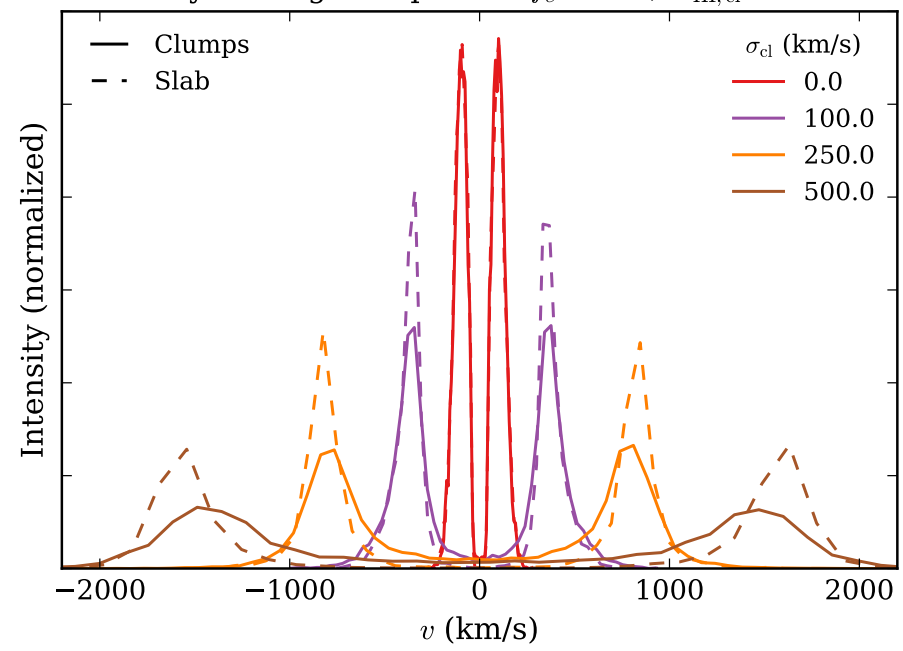

Fig. 5. Solid lines show the Ly $\alpha$ spectra for a constant clump column density $N_{\mathrm{HI}, \mathrm{cl}}=10^{16} \mathrm{~cm}^{-2}$, and covering factor $f_{\mathrm{c}} \approx 1000$. The dashed lines show, for comparison, the spectra obtained from slabs with $T_{\text {eff }}\left(\sigma_{\mathrm{cl}}\right)$.

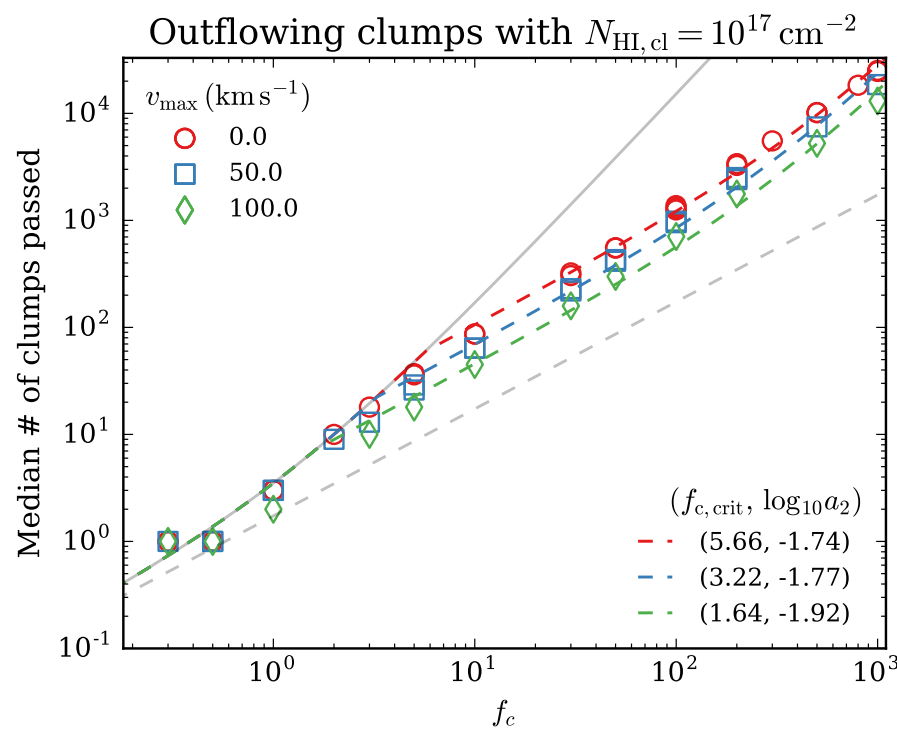

Fig. 6. Number of clumps passed vs. $f_{\mathrm{c}}$ for clumps with $N_{\mathrm{HI}, \mathrm{cl}}=$ $10^{17} \mathrm{~cm}^{-2}$ and outflowing motions with different maxima $v_{\max }$. The dashed lines show fits of Eq. (16) to the data points and the gray solid line shows the limit with $f_{\mathrm{c}, \text { crit }}>10^{3}$.

with the same total column density and velocity structure. We focus on the case with constant clump column density $N_{\mathrm{HI}, \mathrm{cl}}=$ $10^{17} \mathrm{~cm}^{-2}$ and show three cases, $f_{\mathrm{c}}=\{1,100,1000\}$, which match the slab case increasingly well for all four values of $v_{\max }$. This implies that the spectra become more asymmetric as they converge toward the homogeneous limit. The asymmetry develops because the outflow shifts the scattering cross section in the observers reference frame toward the blue. Thus, the optical depth for photons with frequency redward of line center (e.g., the back-scattered photons off the far side of the system) is lowered allowing for easier escape. We discuss the result of higher asymmetry with increased number of clumps further in Sect. 5.

Figure 8 shows this increase in asymmetry with greater $f_{\mathrm{c}}$ for fixed outflow velocities of $v_{\max }=\{100,1000\} \mathrm{km} \mathrm{s}^{-1}$ and total column densities of $N_{\mathrm{HI} \text {,total }}=4 / 3 \times\left\{10^{18}, 10^{19}, 10^{20}\right\} \mathrm{cm}^{-2}$. We characterize the spectral asymmetry by the integrated flux ratio of the blue over the red part of the spectra (minus one), i.e., a value of -1 means that all photons escape redward of line center $(x \leq 0)$ and if this quantity is zero the spectrum is symmetric (around $x=0$ ). While the transition from symmetric to dominantly red spectra for $v_{\max }=100 \mathrm{~km} \mathrm{~s}^{-1}$ is nearly independent of the column density at $f_{\mathrm{c}} \sim 10$, this is not the case for $v_{\max }=1000 \mathrm{~km} \mathrm{~s}^{-1}$ where a larger total column density implies a shift at lower $f_{\mathrm{c}}$. This is because of the dependence of $f_{\mathrm{c} \text {, crit }}$ on $v_{\text {max }}$ and $N_{\mathrm{HI} \text {,total }}$ that is described in Sect. 2.3.4. In particular, as seen in Eq. (13), $f_{\mathrm{c} \text {, crit }}$ does not depend on the column density if $v_{\max }$ is small.

\subsection{Emission within the clumps}

In this section we study the effect of the emission originating from inside the clumps. This case resembles Ly $\alpha$ production due to cooling in the inner parts of the clumps or to recombination events in the outer layer of (self-shielding) clumps caused by an external ionizing source. The latter is sometimes referred to as fluorescence (e.g., Hogan \& Weymann 1987; Mas-Ribas \& Dijkstra 2016). In both cases, Ly $\alpha$ are produced in the reference frame of the clumps and experience an initial optical depth before entering the inter-clump medium; both effects shape the "intrinsic" spectrum.

Figure 9 compares some spectra with starting position inside the clumps to those previously presented, i.e., with starting position at midplane. The clumps in this case possess a column density of $N_{\mathrm{HI}, \mathrm{cl}}=10^{17} \mathrm{~cm}^{-2}$, which means they are optically thick to Ly $\alpha$ radiation. The effect of this can be seen best in the spectrum with $f_{\mathrm{c}}=1$ (red curve in Fig. 9). This contrasts with a double-peaked profile due to the escape from the clump to the single-peaked profile from the random walk process between the clumps. For greater values of $f_{c}$, however, this "initial feature" gets washed out from the scatterings off subsequent clumps and the spectra are independent of the emission site.

In Fig. 10 we show a similar plot for moving clumps. In this case them frequencies of the photons are rescaled according to the value of $\sigma_{\mathrm{cl}}$ for presentation purposes. This means that in the spectra for $\sigma_{\mathrm{cl}}=10^{4} \mathrm{~km} \mathrm{~s}^{-1}$ (shown in purple) are within a full width at half maximum (FWHM) of $\Delta x \sim 5000$ the widest of the presented spectra. As previously stated, for the large $f_{\mathrm{c}} \gg 1$ cases, the spectra with the emission sites within the clumps resemble closely those with emission sites in the midplane. The only difference is that the latter are slightly wider and possess a smaller flux at line center, which is simply because a number of clumps are located at the boundary of the slab. This is encouraging as it shows that our results are more general, that is, not dependent on the exact emission site. However, a small caveat is that for spherical geometries most clumps are located at large radii, which might make this setup more sensitive to in-clump emission; on the other hand, the outermost clumps might emit less Ly $\alpha$ photons as some are "shadowed" by clumps closer to the ionizing source.

\subsection{Dusty clumps}

When placing absorbing dust in the clumps, which we characterize by the all-absorbing dust optical depth $\tau_{\mathrm{cl}, \mathrm{d}}$, Ly $\alpha$ can be destroyed leading to an escape fraction $f_{\text {esc }} \leq 1$. Interestingly, in clumpy medium, the $\operatorname{Ly} \alpha$ escape fraction might be larger than the continuum fraction as predicted by Neufeld (1991). This Neufeld effect occurs because Ly $\alpha$ photons may "surface scatter" off the neutral clumps, thus, effectively shielding the dust 


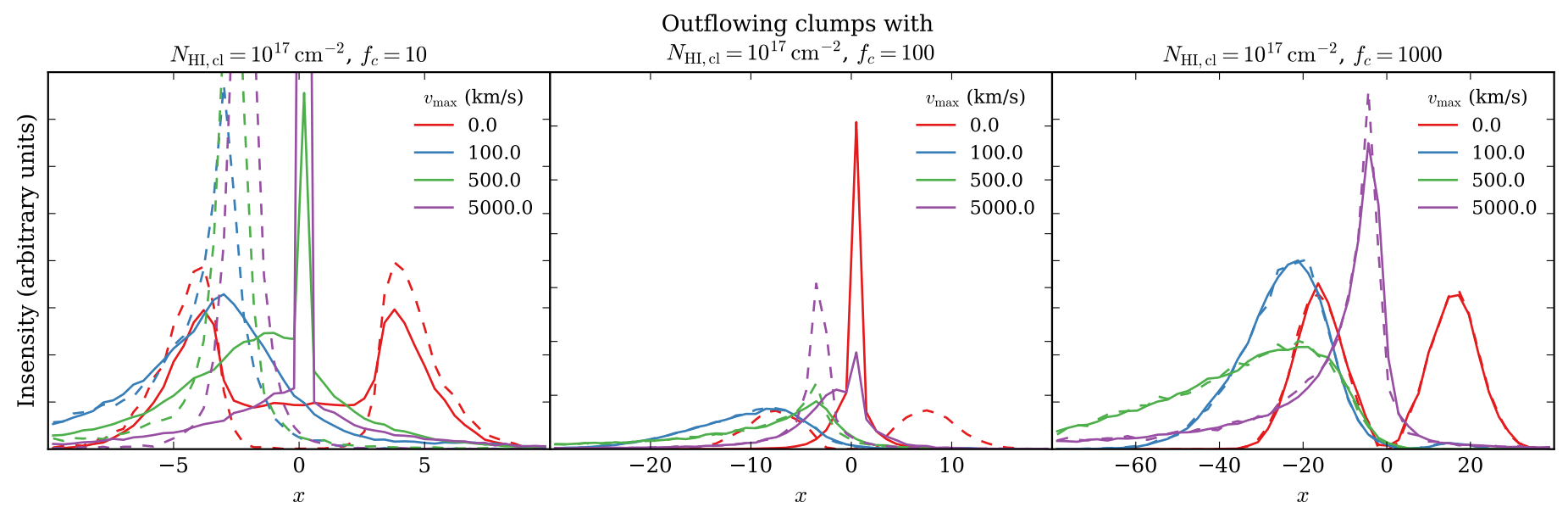

Fig. 7. Ly $\alpha$ spectra using a setup of outflowing clumps with linear velocity profile for $N_{\text {HI,cl }}=10^{17} \mathrm{~cm}^{-2}$ and four different maximal velocities $v_{\max }$. The dashed lines in corresponding colors show the spectra emergent from a slab with the same column density and velocity structure. Each subpanel displays a case with different covering factor corresponding to increasing agreement with the homogeneous setup.

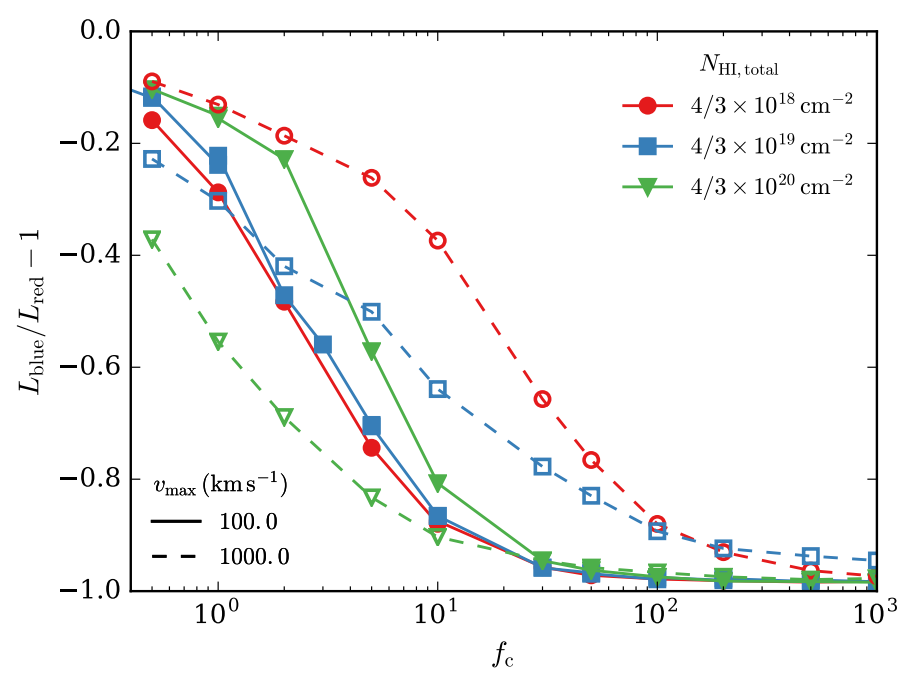

Fig. 8. Integrated blue over integrated red flux (minus one) vs. covering factor for different combinations of $v_{\max }$ and $N_{\mathrm{HI}, \text { total }}$. With increasing $f_{\mathrm{c}}$ the spectra become more redshifted. See Sect. 4.3 for details.

from these clumps. Therefore, one expects the observed Ly $\alpha$ equivalent widths to be potentially much larger than the intrinsic Ly $\alpha$ equivalent widths. Hansen \& Oh (2006) characterized this effect more systematically using Ly $\alpha$ radiative transfer simulations for a wide range of parameters. Building upon their work, Laursen et al. (2013) found, however, that the boosting vanishes in an area of the parameter space that they tried to constrain by observations. Specifically, out of their $4 \times 10^{3}$ models only a few percent showed an equivalent width boost (see also Duval et al. 2014, for a study of the Neufeld effect in clumpy shells). Laursen et al. (2013) thus concluded, "consider the Neufeld model to be an extremely unlikely reason for the observed high equivalent widths (EWs)". All these studies focused on values of $f_{\mathrm{c}} \sim 1$ and we want to revisit Ly $\alpha$ escape in clumpy medium with several orders of magnitude greater covering factors. Thus, it is not entirely clear from the literature whether radiative transfer effects from clumpy media can explain the extreme equivalent width measurements observed in some galaxies. However, Laursen et al. (2013) identified some criteria that have to be fulfilled such as relatively slowly moving clumps with high-dust optical depths.

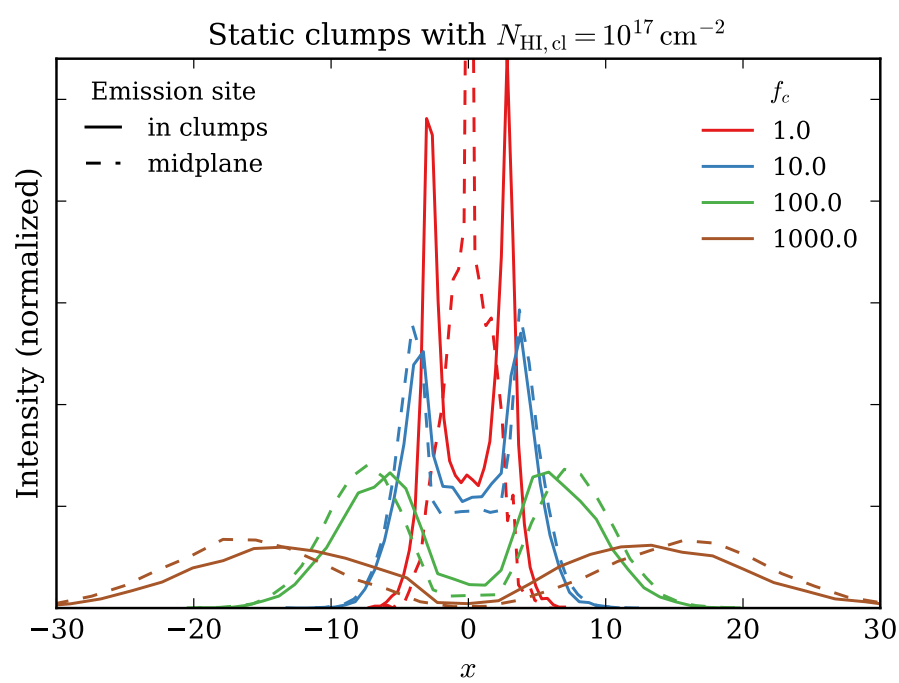

Fig. 9. Ly $\alpha$ spectra for a constant clump column density $N_{\mathrm{HI}, \mathrm{cl}}=$ $10^{17} \mathrm{~cm}^{-2}$ and three values of $f_{\mathrm{c}}$. The solid lines indicate the spectral shape with emission inside the clumps, whereas the dashed lines show, for comparison, the spectra obtained from midplane emission.

Instead of re-running the radiative transfer simulations for various dust contents, we use the information of the hydrogen column density "seen" by each photon package to compute the Ly $\alpha$ escape fraction as in Gronke et al. (2015), which yields an escape fraction for each photon package that is

$f_{\mathrm{esc}, i}=\exp \left[-\frac{\hat{N}_{\mathrm{HI}, i}}{N_{\mathrm{HI}, \mathrm{cl}}} \tau_{\mathrm{d}, \mathrm{cl}}\right]$.

Here, $\hat{N}_{\mathrm{HI}, i}$ is the column density experienced by photon package $i$. Given $f_{\text {esc }, i}$ for a certain setup one can now obtain (a) the overall Ly $\alpha$ escape fraction as the average of $f_{\text {esc, } i}$; and (b) the spectral shape altered through dust by simply assigning each photon package the weight $f_{\text {esc }, i}$ when assembling the spectrum.

In Fig. 11 we plot the Ly $\alpha$ escape fraction versus $f_{\mathrm{c}}$ for a constant total dust and hydrogen number content. A similar trend is visible for all three values of $\tau_{\mathrm{d} \text {,total }}$ shown: with increasing $f_{\mathrm{c}}$, first an approximately linear fall off in escape fraction before a flattening occurs, that is, $f_{\text {esc }} \sim$ const. for $f_{\mathrm{c}} \gtrsim 40$. Interestingly, the position of this threshold is independent of $\tau_{\mathrm{d} \text {,total }}$, which 


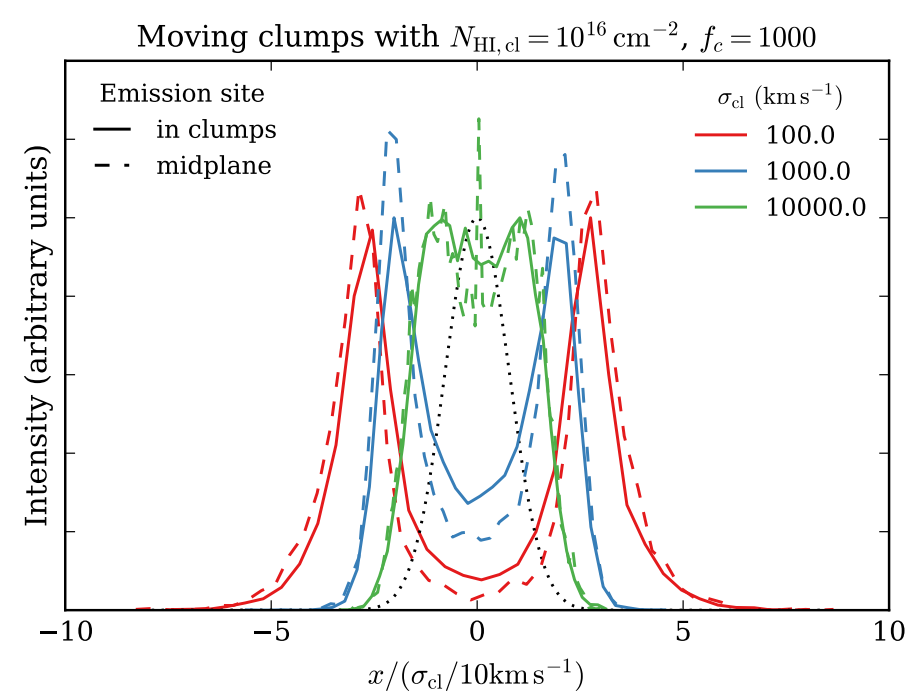

Fig. 10. Ly $\alpha$ spectra for a constant geometry with $N_{\mathrm{HI}, \mathrm{cl}}=10^{16} \mathrm{~cm}^{-2}$, $f_{\mathrm{c}}=1000$ and three values of $\sigma_{\mathrm{cl}}$. The solid lines indicate the spectral shape with emission inside the clumps, whereas the dashed lines show, for comparison, the spectra obtained from midplane emission. For presentation purposes we rescaled the $x$-axis according to the value of $\sigma_{\mathrm{cl}}$. The black dotted line shows the intrinsic spectrum, which has the same width for all $\sigma_{\mathrm{cl}}$ owing to the rescaling.

hints at an origin in the nature of the radiative transfer. The flattening occurs at the boundary between the "free streaming" and "homogeneous" regime because in the former the probability of absorption is proportional to the number of clump interactions (and, thus, $f_{\mathrm{c}}$ ), whereas in the latter the escape fraction is set by the total dust content only and does not grow further with $f_{\mathrm{c}}$. We discuss this phenomenon in more detail in Sect. 5.2.

An implication of the respective escape fractions of the two regimes is visible in Fig. 12. Here we show several values of $N_{\mathrm{HI}, \mathrm{cl}}$ for the static setup using $\tau_{\mathrm{d}, \mathrm{cl}}=10^{-4}$ (empty symbols) and $\tau_{\mathrm{d}, \mathrm{cl}}=1$ (filled symbols), which correspond to metallicities of $Z / Z_{\odot}=0.63\left(\tau_{\mathrm{d}} / 10^{-4}\right)\left(10^{17} \mathrm{~cm}^{-2} / N_{\mathrm{HI}, \mathrm{cl}}\right)($ Pei 1992; Laursen et al. 2009); this reaches clearly unrealistic values. However, as in this paper we are interested in the fundamental impact of the individual parameters, we also study these extreme values. Also shown in Fig. 12 (with a black [gray] solid line for the low [high] dust content) is the proposed analytic solution for $f_{\mathrm{esc}}$ by Hansen \& Oh (2006)

$f_{\mathrm{esc}}^{\mathrm{HO} 06}=1 / \cosh \left(\sqrt{2 N_{\mathrm{cl}} \epsilon}\right)$,

where for $N_{\mathrm{cl}}$ we used Eq. (8) (with $\left(a_{1}, b_{1}\right)=(3 / 2,2)$ as found in Sect. 4.1) and for the clump albedo (i.e., the fraction of incoming photons that are reflected) $\epsilon$, we adopted a value of $c_{1}\left(1-\mathrm{e}^{-\tau_{\mathrm{d}, \mathrm{cl}}}\right)$ with $c_{1}=1.6\left[c_{1}=0.06\right]$ to match the $N_{\mathrm{HI}, \mathrm{cl}}=10^{22} \mathrm{~cm}^{-2}$ data points for $\tau_{\mathrm{d}, \mathrm{cl}}=10^{-4}\left[\tau_{\mathrm{d}, \mathrm{cl}}=1\right]$. The behavior for the low- and high-dust contents is quite different. On the one hand, the escape fractions versus $N_{\mathrm{HI}, \mathrm{cl}}$ scales for $\tau_{\mathrm{d}, \mathrm{cl}}=1$ (filled symbols in Fig. 12) as predicted by Hansen \& Oh (2006) in their "surface scatter" approximation, that is, a larger clump hydrogen column density "shields" the dust better from the Ly $\alpha$ photons and thus prevents their destruction more efficiently. On the other hand, however, this is not the case for the low-dust scenario presented in Fig. 12 (with unfilled symbols) where a larger value of $N_{\mathrm{HI}, \mathrm{cl}}$ implies a lower $f_{\text {esc }}$. This is because here the dust optical depth through all the clumps (shown in the black dotted line in Fig. 12) is lower than the accumulated

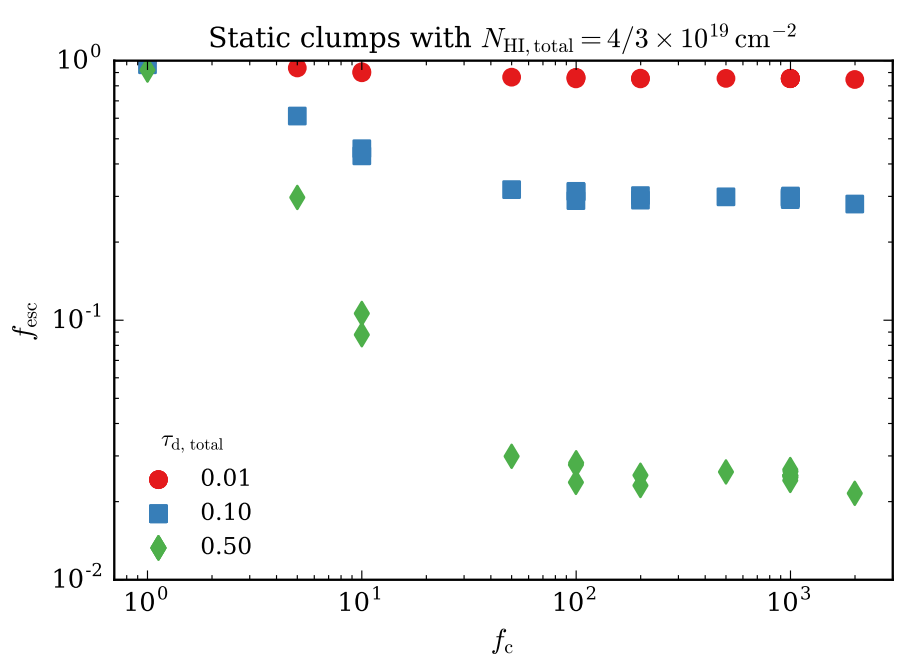

Fig. 11. Ly $\alpha$ escape fraction vs. $f_{\mathrm{c}}$ for a fixed total hydrogen column density $N_{\mathrm{HI}, \text { total }}$ and dust optical depth $\tau_{\mathrm{d} \text {,total }}$.

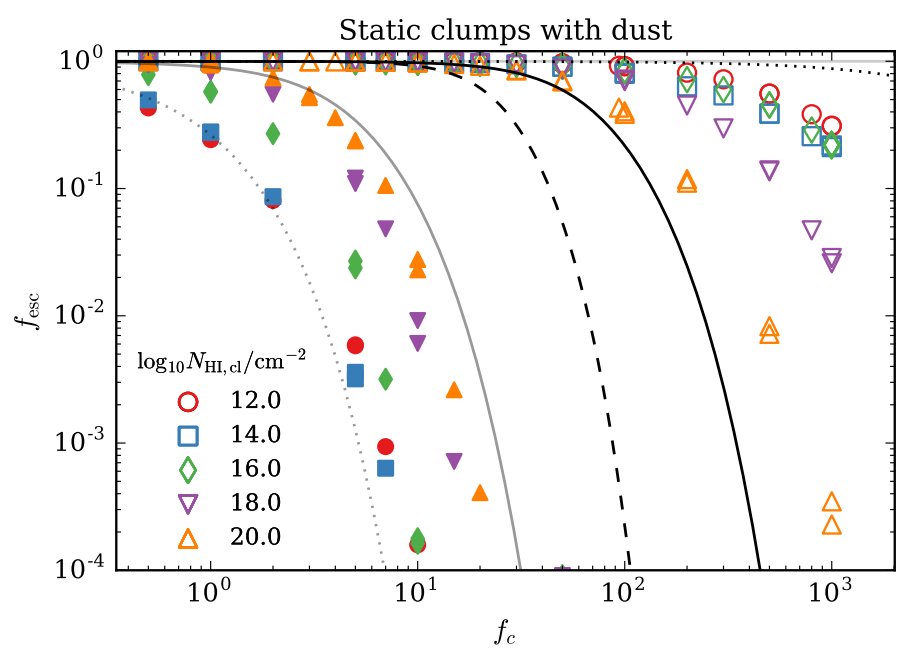

Fig. 12. Ly $\alpha$ escape fraction vs. $f_{\mathrm{c}}$ for various values of $N_{\mathrm{HI}, \mathrm{cl}}, \tau_{\mathrm{d}, \mathrm{cl}}=1$ and $\tau_{\mathrm{d}, \mathrm{cl}}=10^{-4}$ (filled and unfilled symbols, respectively). The black (gray) curves show some analytic curves for $\tau_{\mathrm{d}, \mathrm{cl}}=10^{-4}\left[\tau_{\mathrm{d}, \mathrm{cl}}=1\right]$. The solid curve shows the Hansen \& Oh (2006) formula as given by Eq. (18), the dashed line is the escape fraction from a homogeneous slab with $N_{\mathrm{HI}}=4 / 3 f_{\mathrm{c}} 10^{22} \mathrm{~cm}^{-2}$ as given by Neufeld (1990), and the dotted line is simply $\exp \left(-4 / 3 f_{\mathrm{c}} \tau_{\mathrm{d}, \mathrm{cl}}\right)$, symbolizing a continuum escape fraction.

dust optical depth through the subsequent random-walk clump encounters (black solid line), i.e., $\exp \left(-4 / 3 f_{\mathrm{c}} \tau_{\mathrm{d}, \mathrm{cl}}\right) \lesssim f_{\mathrm{esc}}^{\mathrm{HO}}$. Consequently, configurations in the "free-streaming" regime can possess enhanced Ly $\alpha$ escape fractions compared to the "random walk" regime (see Sect. 5.2 for a more detailed discussion). Still, both cases possess (much) larger escape fractions than a homogeneous slab, which is shown in Fig. 12 with a black dashed line. Here, we use the derived escape fraction by Neufeld (1990) with $N_{\mathrm{HI}}=4 / 3 \times f_{\mathrm{c}} 10^{22} \mathrm{~cm}^{-2}$ and $\tau_{\mathrm{d}}=4 / 3 f_{\mathrm{c}} \tau_{\mathrm{d}, \mathrm{cl}}$, i.e., with equal column densities as in the $N_{\mathrm{HI}, \mathrm{cl}}=10^{22} \mathrm{~cm}^{-2}$ case.

The same quantity, i.e., $f_{\text {esc }}$ versus $f_{\mathrm{c}}$, for the case of randomly moving clumps is plotted in Fig. 13. As previously, the escape fraction departures from the curve given by Eq. (18) for $f_{\mathrm{c}} \gtrsim f_{\mathrm{c} \text {, crit. }}$. The lower number of clump encounters in this regime leads to a significantly higher escape fraction, for example, $f_{\mathrm{esc}} \sim 10^{-1}$ for $\sigma_{\mathrm{cl}}=500 \mathrm{~km} \mathrm{~s}^{-1}$. 


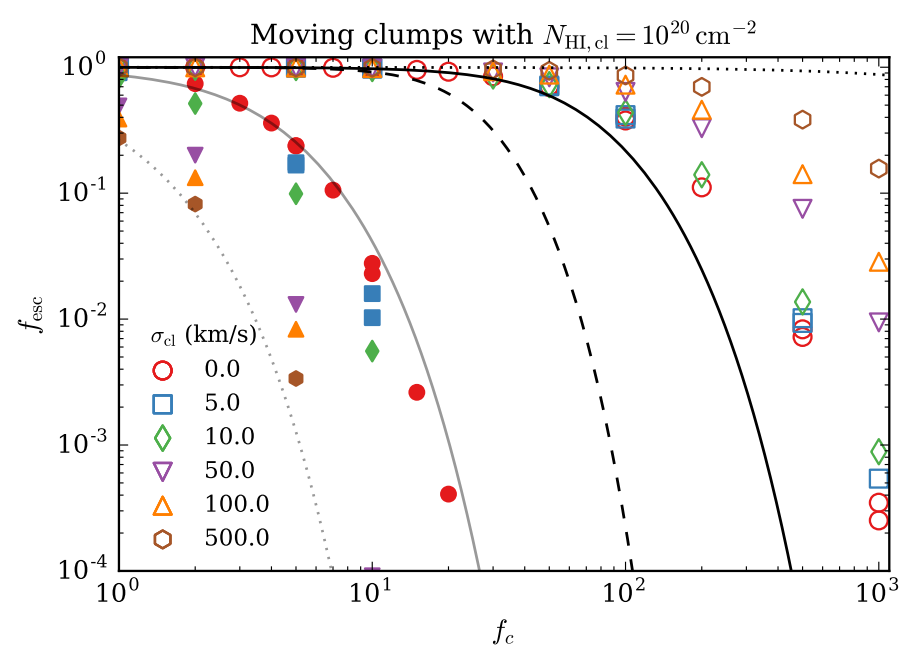

Fig. 13. Ly $\alpha$ escape fraction vs. $f_{\mathrm{c}}$ for fixed clumps with $\tau_{\mathrm{d}, \mathrm{cl}}=10^{-4}$, $N_{\mathrm{HI}, \mathrm{cl}}=10^{20} \mathrm{~cm}^{-2}$ and various values of $\sigma_{\mathrm{cl}}$. The curves are the same as in Fig. 12 for comparison.

\section{Discussion}

In this section, we discuss our results in light of the various escape regimes discussed in Sect. 2 (Sect. 5.1). Furthermore, we analyze what implications our results have for "Ly $\alpha$ equivalent width boosting" (in Sect. 5.2), and make the connection to observational results (of "shell-model" fitting; Sect. 5.3) and to radiative transfer results through hydrodynamical simulations (Sect. 5.4).

\subsection{Regimes of the clumpy model}

Figure 14 summarizes our findings for the static case. Here, color shows the flux at line center expressed in units of flux at the peak of the spectra $F(x=0) / F_{\text {peak }}$. This measure is $\sim 1$ for a singlepeaked spectra and is less for double-peaked spectra; a value of $\sim 0$ corresponds to an optically thick, slab-like spectrum. We highlighted the dividing value of $F(x=0) / F_{\text {peak }}=1 / 2$ specifically.

Also visible in Fig. 14 are the three regimes described Sect. 2, along with our analytic estimates. They can be summarized as follows:

- Optically thin regime. For an overall optical depth, $\tau_{0, \text { total }}=$ $4 / 3 f_{\mathrm{c}} \tau_{0, \mathrm{cl}} \lesssim 1$, the $N_{\mathrm{cl}}-f_{\mathrm{c}}$ scaling is shallow, and the emergent spectra are single peaked. The dotted line in Fig. 14 indicates this boundary.

- Homogeneous regime. If not in the "optically thin regime", for $f_{\mathrm{c}} \gtrsim f_{\mathrm{c} \text {,crit }}$ we also found a shallower $N_{\mathrm{cl}}-f_{\mathrm{c}}$ scaling than Hansen \& Oh (2006). This is because of the preferential escape in an optically thick medium through single excursion, which causes broad, double-peaked spectra. The dashed line in Fig. 14 indicates $f_{\text {c,crit }}$ as a function of $N_{\mathrm{HI}, \mathrm{cl}}$. Above this line we find $F(x=0) / F_{\text {peak }} \rightarrow 0$ denoting double-peaked spectra as predicted. Similarly, below this line the numerical results show single-peaked spectra.

- Random-walk regime. For optically thick clumps and $f_{\mathrm{c}} \lesssim$ $f_{\mathrm{c} \text {, crit }}$ we recovered the results of Hansen \& Oh (2006), i.e., $N_{\mathrm{cl}} \propto f_{\mathrm{c}}{ }^{2}$ and single-peaked spectra owing to a surfacescattering escape of the photons.

As noted in Sect. 2.3.3 these regimes break down for $f_{\mathrm{c}} \lesssim$ 3 , which is an area of the parameter space that was previously by studied by Hansen \& Oh (2006), Laursen et al. (2013),

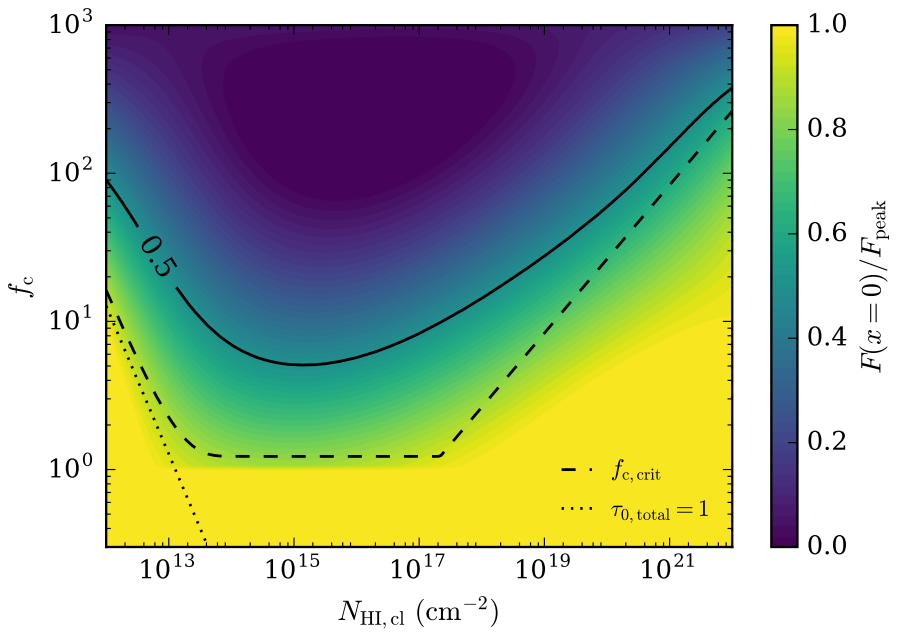

Fig. 14. Overview of the different regimes for the static $\left(\sigma_{\mathrm{cl}}=v_{\max }=0\right)$ setup. The color coding shows our (interpolated) numerical results in terms of the flux at line center divided by the peak flux of the spectrum, i.e., a value of $\sim 0[\sim 1]$ quantifies a double- [single-]peaked spectrum. Specifically this quantity is $1 / 2$ at the solid line. The dashed line indicates the $f_{\mathrm{c} \text {, crit }}$ (Eq. (10)), and the dotted line is the boundary to the low-density regime $\left(\tau_{0, \text { total }}=1\right)$.

Gronke \& Dijkstra (2016), where the probability of not finding a clump in a certain sightline is non-negligible (this allows for nonzero ionizing photon escape fraction; see Dijkstra et al. 2016).

Figure 15 shows the transition from double- to single-peaked spectra for randomly moving clumps. The color coding shows in this case the peak position of the spectrum, where white is $x_{\text {peak }} \sim 3$, that is, when the peak position moves outside the core of the line ${ }^{7}$. For faster clumps, this boundary moves to greater values of $f_{\mathrm{c}}$, making it more likely to obtain a single-peaked spectrum (at line center). The black dashed lines in Fig. 15 denote $f_{\mathrm{c} \text {, crit }}$ from Eq. $(12)^{8}$; in other words below this line the velocity space is not well sampled and allows photons at line center to escape.

The same line is also indicated in Fig. 16, where we focus on the clump column density $N_{\mathrm{HI}, \mathrm{cl}}=10^{17} \mathrm{~cm}^{-2}$ as predicted by "shattering" (McCourt et al. 2016). Here the peak position (in $\log$ scale) is color coded as a function of covering factor and clump velocity dispersion. For large values of $\sigma_{\mathrm{cl}}$, the transition to double-peaked spectra occurs at a larger covering fraction, since more clumps are required to sample the broader velocity distribution. Below this threshold, we see a single-peaked spectrum from photons that escape through holes in velocity space.

Figure 17 shows this increase in asymmetry with greater $f_{\mathrm{c}}$ for fixed outflow velocity and total column density of $N_{\mathrm{HI} \text {,total }}=$ $4 / 3 \times 10^{19} \mathrm{~cm}^{-2}$. Here, the color corresponds to the asymmetry of the spectra, which we define as in Sect. 4.3 to be the ratio of the integrated blue over the red flux minus one. In Fig. 17 we also indicate graphically the conditions for homogeneous escape discussed in Sect. 2.3.4, that is, that the adjacent clump is optically thick $\left(4 / 3 \tau_{\mathrm{cl}}\left(x_{\text {next }}\right) \gtrsim 1\right.$ with $\left.x_{\text {next }} \equiv v_{\text {max }} /\left(f_{\mathrm{c}} v_{\text {th }}\right)\right)$, and that

\footnotetext{
7 We used an alternative criterion because for larger $\sigma_{\mathrm{cl}}$ the spectra can be very broad, and thus $F(x=0) / F_{\text {peak }}$ becomes noise dominated. However, both measures can be used to distinguish between single- and double-peaked spectra.

8 In fact, we used the exact functional form for $\sigma_{\mathrm{HI}}(x)$ and did not resort to the approximation as in Eq. (12), which yields a slightly better fit to the data.
} 

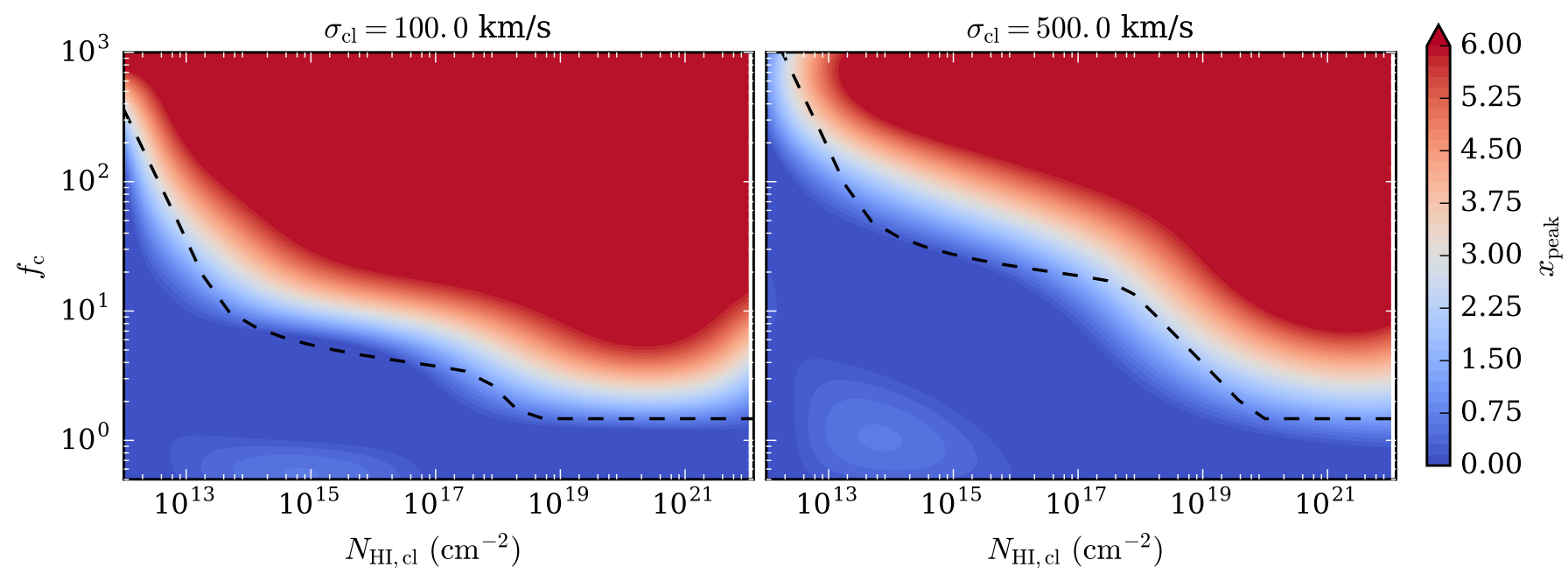

Fig. 15. Overview of the $f_{\mathrm{c}}-N_{\mathrm{HI}, \mathrm{cl}}$-plane with moving clumps for two different values of $\sigma_{\mathrm{cl}}$. The color coding shows the spectral peak position $x_{\text {peak }}$ (truncated at $x_{\text {peak }}=6$ ). The dashed lines show $f_{\text {c, crit }}$ in the moving case (Sect. 2.3.4), i.e., below this line the velocity distribution of clumps is not sampled well.

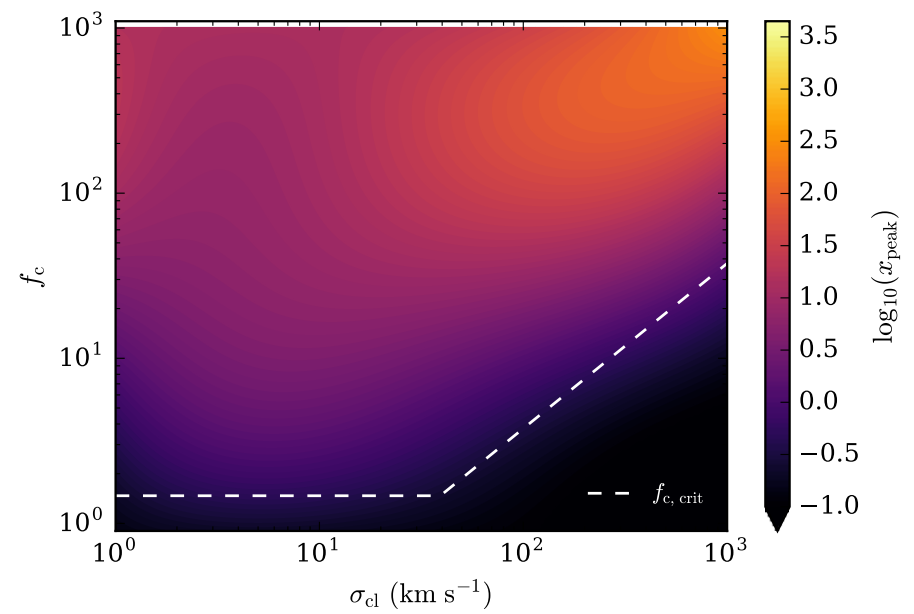

Fig. 16. Overview of the spectral shape for $N_{\mathrm{HI}, \mathrm{cl}}=10^{17} \mathrm{~cm}^{-2}$ (the clump column density predicted by McCourt et al. 2016). The color coding denotes (the $\log$ of) the peak position $x_{\text {peak }}$, i.e., low values (in black) represent a single-peaked feature. The white dashed line is again the $f_{\mathrm{c} \text {, crit }}$ boundary in the moving case.

the initial scatterings occur in the core of the line $\left(x_{\text {next }}<x_{*}\right)$. If both conditions are fulfilled (and sufficient outflows are present, i.e., $v_{\max } \gtrsim 50 \mathrm{~km} \mathrm{~s}^{-1}$ ), the emergent spectrum is asymmetric toward the red side (as visible from the red region in Fig. 17).

\subsection{Escape fractions and equivalent width boosting}

The different regimes for Ly $\alpha$ radiative transfer through a multiphase gas have different implications for the Ly $\alpha$ escape fraction when dust is present within the clumps.

- Inside the "optically thin regime" $\left(\tau_{0, \text { total }} \lesssim 1\right)$, the Ly $\alpha$ escape fraction is equal to the continuum escape fraction as Ly $\alpha$ photons stream through all the clumps and are affected by the dust content within these clumps. Hence, $f_{\mathrm{esc}} \approx$ $\exp \left(-\tau_{\text {d,total }}\right)$. This can be seen for the $N_{\mathrm{HI}, \mathrm{cl}} \lesssim 10^{14} \mathrm{~cm}^{-2}$, $f_{\mathrm{c}} \lesssim f_{\mathrm{c}, \text { crit }}$ data points in Fig. 12.

- In the "random-walk regime", we confirm the escape fraction given by Hansen \& Oh (2006, see Eq. (18), apart from geometrical pre-factors). In this regime the governing quantity for the escape fraction is $\epsilon$, i.e., the absorption probability per clump interaction and the number of clumps encountered $N_{\mathrm{cl}}$. In this regime, the latter is merely a function of $f_{\mathrm{c}}$ (see Eq. (8)), but $\epsilon$ depends non-trivially on $N_{\mathrm{HI}, \mathrm{cl}}$ and the clump movement as a result of variations in how deep the photons penetrate into the clumps. This is why there is some scatter in $f_{\text {esc }}$ in this regime; this is visible, for instance, in Fig. 12. This is the only regime in which $\operatorname{Ly} \alpha$ photons are shielded from dust, thus, allowing for "equivalent-widthboosting" (Hansen \& Oh 2006). That is, the ratio between the Ly $\alpha$ and UV escape fraction might be greater than unity.

- Finally, in the "homogeneous regime", the behavior is a combination of the above two behaviors. Initially, the photons (on average) interacts with $\sim N_{\mathrm{cl}}\left(f_{\mathrm{c} \text {, crit }}\right)$ clumps before diffusing to the line wings and escaping through free-streaming, which leads to other $\sim f_{\mathrm{c}}$ clump encounters (cf. Fig. 2). Consequently, in this regime the escape fraction is approximately given by $f_{\text {esc }} \sim f_{\text {esc }}^{\mathrm{HO} 06}\left(f_{\mathrm{c} \text {, crit }}\right) \mathrm{e}^{-\tau_{\mathrm{d}, \text { total }}}$. This causes the flattening of $f_{\text {esc }}$ versus $f_{\mathrm{c}}$ in Fig. 11 as in this case $\tau_{\mathrm{d} \text {,total }}$ is kept constant.

From the above considerations, one can see that the escape fraction depends on several parameters and is therefore nontrivial to predict. As a consequence, in Sect. 4.5 we demonstrated that the Ly $\alpha$ escape fraction may either increase or decrease with increasing metallicity, which is $Z \propto \tau_{\mathrm{d}} / n_{\mathrm{HI}}$ (Pei 1992; Laursen et al. 2009), depending on the dust optical depth through an individual clump $\tau_{\mathrm{d}, \mathrm{cl}}$ (see the trends in the filled and unfilled symbols in Figs. 12 and 13). The controlling parameter is essentially the ratio of absorption probability per surface scatter to the absorption probability per clump passing. Moreover, we have shown that Ly $\alpha$ escape fractions can be large, even for large values of $f_{\mathrm{c}}$. Thus, we find that homogeneous, "slab-like" spectra can be observable even in models with significant dust content (as is realistic; see Sects. 5.3 and 5.4).

Regarding the equivalent width boosting we found, one necessary requirement for the "Neufeld effect" to be active is that Ly $\alpha$ photons escape via surface scatterings off the clumps, i.e., in the "random walk" regime. This implies that the emergent spectrum is narrow and single peaked at line center (as already noted 
by Laursen et al. 2013), which is a clear observational signature for equivalent width boosting to be active ${ }^{9}$.

\subsection{Connection to a homogeneous medium}

Observed Ly $\alpha$ spectra can often be successfully modeled using a simple model called the shell model (see, for instance, Hashimoto et al. 2015; Karman et al. 2017). This shell model consists of a central Ly $\alpha$ (and continuum) emitting region that is surrounded by a moving shell of hydrogen and dust (Ahn et al. 2003; Verhamme et al. 2006). It is somewhat surprising that this simple, six-parameter model can account for the likely radiative transfer effects happening in the complex, multiphase medium of a variety of galaxies and their environments. Since the shell model is clearly very idealized, it is unclear what the extracted shell-model parameters mean physically. In Gronke \& Dijkstra (2016) we found that a simple one-to-one mapping between the shell-model parameters and those from a clumpy medium is not possible; for the most part, the shell model cannot reproduce the spectra emergent from a multiphase medium. This failure mostly results from the high fluxes at line center from the multiphase simulations, which are hard to obtain through radiative transfer through a uniform gas distribution (such as a shell).

However, in Gronke \& Dijkstra (2016) we restricted our analysis to covering factors of $f_{\mathrm{c}} \sim O(1)$ (and $\sigma_{\mathrm{cl}} \lesssim 100 \mathrm{~km} \mathrm{~s}^{-1}$ ), i.e., the "random-walk" and "optically thin" regime. As we showed here, for a (much) greater number of clouds the system approaches a slab-like state; this state leads to, for example, much lower fluxes at line center for the resulting Ly $\alpha$ spectrum. Hence, these multiphase spectra might be closer to observed spectra. Whether or not the shell-model parameters correspond to physical parameters of a clumpy medium with large $f_{\mathrm{c}}$ will be analyzed for a future work. However, our results show that for $f_{\mathrm{c}} \gtrsim f_{\mathrm{c} \text {, crit }}$ the spectra are similar to a slab with the same column density. Furthermore, we fitted shellmodels to three spectra originating from a clumpy medium with $N_{\mathrm{HI}, \text { total }}=4 / 3 \times 10^{19} \mathrm{~cm}^{-2}, v_{\max }=50 \mathrm{~km} \mathrm{~s}^{-1}$ and various covering factors. The column density of our system and the outflow velocity were chosen to be well within the range of shell-model parameters recovered from observations (e.g., Yang et al. 2017). Prior to fitting, we smoothed the spectra using a Gaussian kernel with FWHM $W \sim 24 \mathrm{~km} \mathrm{~s}^{-1}$. Figure 18 shows the three spectra and the best-fit shell model spectra. The resulting shell-model parameters are also shown in the figure. While the fits for $f_{\mathrm{c}}=3$ and $f_{\mathrm{c}}=10$ are rather poor and the recovered shell-model column densities are more than an order of magnitude off, the spectra for $f_{\mathrm{c}}=50$ can be remarkably well recovered. Interestingly, here the shell column density is very close to the input value, and the recovered shell of $v_{\exp } \approx 25 \mathrm{~km} \mathrm{~s}^{-1}$ outflow velocity corresponds to the mass weighted mean of the used Hubble-like outflow. Also, the dust content and, to some extent, the temperature of the gas are recovered. On the other hand, as the photons are injected at line center, the recovered widths of the intrinsic spectra $\left(\sigma_{\mathrm{i}}\right)$ are too large. This may be to compensate for the narrow coverage of the shell in velocity space. A similar discrepancy in the intrinsic profile width is also found in the literature (e.g., Yang et al. 2016, by comparing $\sigma_{\mathrm{i}}$ with the width of the $\mathrm{H} \alpha$ line), in which this discrepancy might also originate from radiative transfer effects.

\footnotetext{
9 However, if the line is narrow and concentrated on line center then the IGM can suppress the flux because this is where we expect the IGM opacity to peak (see, e.g., Laursen et al. 2011).
}

\begin{tabular}{cccccc}
\hline-1.0 & 1 & 1 & 1 & 1 \\
(redshifted) & -0.8 & -0.6 & -0.4 & -0.2 & -0.0 \\
& & & $L_{\text {blue }} / L_{\text {red }}-1$ & & (symmetric)
\end{tabular}

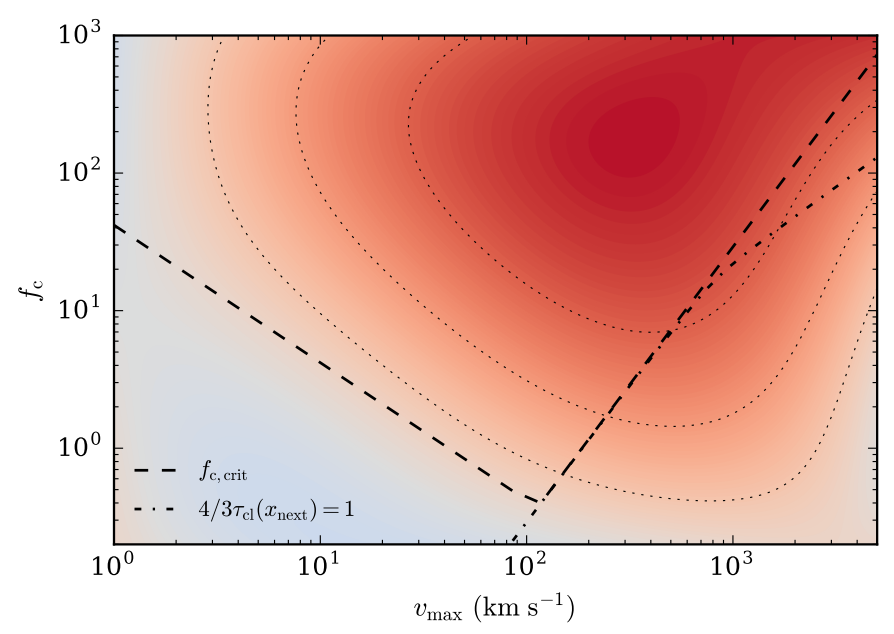

Fig. 17. Asymmetry of the spectra (color coded) as a function of outflow velocity, $v_{\max }$, and covering factor, $f_{\mathrm{c}}$, for a fixed total column density of $N_{\mathrm{HI}, \text { total }}=4 / 3 \times 10^{19} \mathrm{~cm}^{-2}$. As tracer of the asymmetry we chose to display the ratio of the integrated flux on the blue side $(x \geq 0)$ of the line $L_{\text {blue }}$ over the integrated red flux $L_{\text {red }}$ minus one. This implies that a value of 0 (in white) corresponds to a symmetric spectrum, whereas -1 (in dark red) corresponds to a spectrum in which all flux is redward of line center. The contour lines highlight values of $(-0.75,-0.5,-0.25)$. Also shown are the $f_{\mathrm{c} \text {, crit }}$ boundary (Eq. (13)) and the more precise $4 / 3 \tau_{\mathrm{cl}}\left(x_{\text {next }}\right)=1$ deviation. The relatively low values of $f_{\mathrm{c} \text {, crit }}$ imply that large covering factors as predicted by McCourt et al. (2016) lead to asymmetric spectra.

All these points suggest that at least some of the shell-model parameters might have a true physical meaning. In this work, we provide an equally simple but physically meaningful model that serves as a theoretical justification for the shell model. The full mapping from the shell model to the parameters of a multiphase medium with large $f_{\mathrm{c}}$ will be part of future work. However, from our single example it is already apparent that if an observed Ly $\alpha$ spectra can be modeled using a simple, homogeneous shell, one can think instead about a fog of droplets (with $f_{\mathrm{c}} \gg f_{\mathrm{c} \text {, crit }}$ ), which is more realistic given our knowledge about gas properties.

\subsection{Implications for ab initio $L y \alpha$ radiative transfer simulations}

Our findings suggest a possible cause for the mismatch between observed Ly $\alpha$ spectra and those computed with snapshots of hydrodynamical simulations as input; these are sometimes referred to ab initio Ly $\alpha$ radiative transfer simulations.

Observed Ly $\alpha$ spectra from $z \sim 0$ to higher redshifts show several common features:

- A significant shift redward of the main emitting peak. For instance, at $z \sim 2-3$ galaxies selected owing to their strong Ly $\alpha$ emission and dropout-selected galaxies (Ly $\alpha$ emitters and Lyman-break galaxies or LAEs and LBGs, respectively) show shifts of several hundred $\mathrm{km} \mathrm{s}^{-1}$ (e.g., Steidel et al. 2010; Kulas et al. 2012; Erb et al. 2014; Song et al. 2014; Trainor et al. 2015; Hashimoto et al. 2015).

- Asymmetric profiles with mostly stronger red than blue components. For instance, Erb et al. (2014) measured the median 


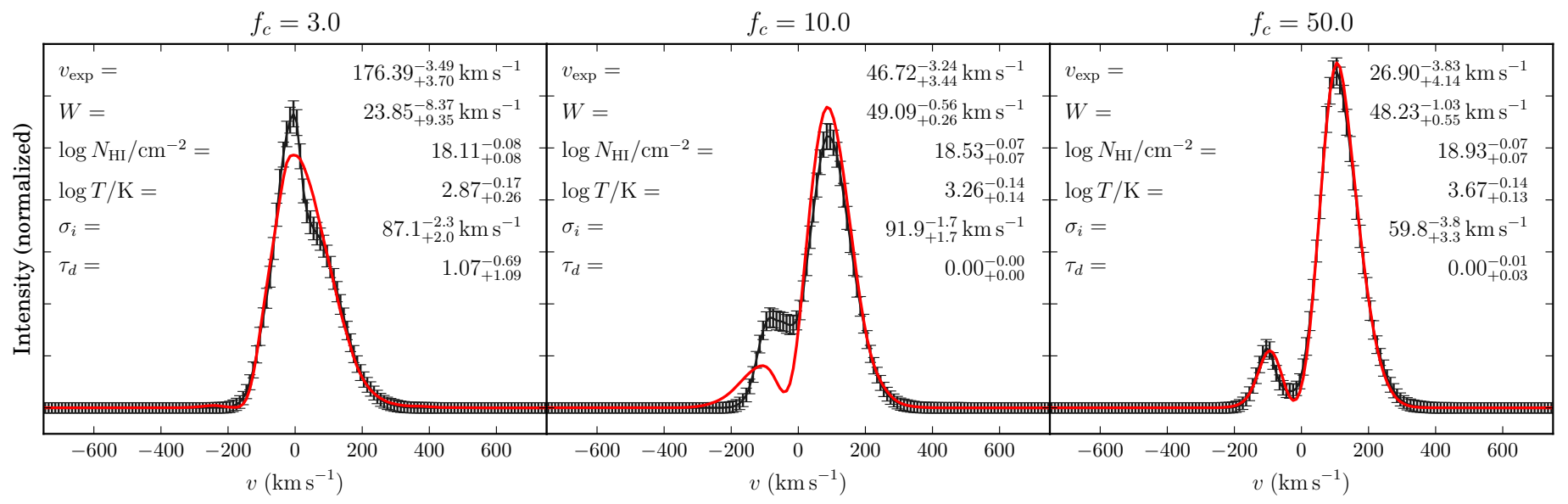

Fig. 18. Fitting shell models to spectra of $\log N_{\mathrm{HI}, \text { total }} / \mathrm{cm}^{-2} \approx 19.1$ and $v_{\max }=50 \mathrm{~km} \mathrm{~s}^{-1}$ and covering factors of $f_{\mathrm{c}}=\{3,10,50\}$ (left to right panel). The black points and red lines show the spectra of the multiphase media and the best-fit shell-model spectra. See Sect. 5.3 for details.

equivalent width ratio $W_{\text {blue }} / W_{\text {red }}$ in their sample of 36 LAEs at $z \sim 2-3$ to be $\sim 0.4$. This is consistent with the findings at $z \sim 0.2$, which also show a dominant red side (by a factor of a few, Henry et al. 2015; Yang et al. 2016, 2017).

- There are roughly as many single as double-peaked spectra. For instance, in the Ly $\alpha$ selected galaxy sample presented by Trainor et al. (2015) of 318 LAEs at $z \sim 2.5-$ $3,41 \%$ show a double-peaked spectra. This fraction agrees well with the double-peaked fraction of $45 \%$ they found in the KBSS-MOSFIRE LBG sample (Steidel et al. 2014) and those of other studies (e.g., Kulas et al. 2012; Yamada et al. 2012 , found ratios of $\sim 1 / 3$ and $1 / 2$, respectively). Only a small part of the double-peaked profiles show a dominant blue peak, which agrees with flux ratio discussed above. Trainor et al. (2015) have quantified these to be $\sim 10 \%$ of the double-peaked spectra.

- For double-peaked spectra, the flux in the "valley" between the peaks is small. Because of smoothing and resolution effects due to the observational aperture, measuring this quantity is challenging, in particular for higher redshifts. However, at lower redshifts Yang et al. (2016) have found in their sample of 12 galaxies at $z \sim 0.2$ the flux ratio between the valley and the red peak to be $0.03_{-0.02}^{+0.08}$ and never greater than 0.27 . Also the 14 galaxies of the "Lyman- $\alpha$ reference sample" (LARS; Östlin et al. 2014) at $0.02<z<0.2$ have a flux ratio between the maximum and minimum of $<0.1$, mostly even consistent with zero (Rivera-Thorsen et al. 2015).

These findings seem to be in stark contrast to the Ly $\alpha$ radiative transfer simulations that use a snapshot of a (highresolution) hydro-dynamical simulation of a galaxy as input geometry (e.g., Tasitsiomi 2006; Laursen \& Sommer-Larsen 2007; Zheng et al. 2010; Barnes et al. 2011; Verhamme et al. 2012; Behrens \& Braun 2014; Smith et al. 2015; Trebitsch et al. 2016). Owing to the computational cost and probable directional dependence of the emergent spectrum (Verhamme et al. 2012; Behrens \& Braun 2014), no statistical compilation of simulation-based spectra has yet been assembled. However, existing predicted spectra are generally too symmetric and/or possess a flux that is too high at line center. This is commonly attributed to (i) CGM in combination with instrumental effects (as discussed in Gronke \& Dijkstra 2016); (ii) radiative transfer effects in close proximity to the origin of the photon; and/or (iii) IGM absorption (Dijkstra et al. 2007; Laursen et al. 2011). All these arguments move the problem to different spatial location (in case of (ii) even to a subgrid scale). However, the last solution cannot be universally invoked, especially at lower redshifts. For instance, Laursen et al. (2011) found that for $z \lesssim 3.5$ only $\lesssim 30 \%$ of the sightlines show a full absorption feature, which would lead to a low flux in the "valley". Furthermore, while the IGM opacity increases with redshift, we see that the $\operatorname{Ly} \alpha$ escape fraction from star-forming galaxies also increases with redshift (Hayes et al. 2011; Blanc et al. 2011; Dijkstra \& Jeeson-Daniel 2013). Both arguments strongly suggest that IGM absorption cannot be the dominant mechanism regulating the visibility of $\operatorname{Ly} \alpha$ emission.

We have shown here that this discrepancy between observations and simulations can be understood easily. Simulations with Lagrangian-type techniques such as adaptive-meshrefinement or smooth-particle-hydrodynamics (AMR and SPH, respectively) reach their highest resolution in the densest regions such as the midplane of the galaxy disk. While future simulations likely reach peak resolutions approaching the $\sim 0.1 \mathrm{pc}$ scale we expect, this still does not capture clump formation and evolution at large distances in the CGM, where the density and resolution remains low. This means the clumps are unresolved and, thus, the covering factor per resolution element is less than unity compared to potentially hundreds as suggested theoretically by McCourt et al. (2016). This lower $f_{\text {c }}$ (while keeping the column density and global structure unchanged) leads to a higher flux at line center (as shown in Fig. 3), less asymmetric spectra (Fig. 17), and in general more "unrealistic spectra" (cf. Gronke \& Dijkstra 2016). Therefore, small-scale structure in the CGM is crucial for modeling radiative transfer through the galaxy. We expect that direct simulation of the multiphase CGM is essentially impossible precisely because it requires very high spatial resolution, even in parts of the galaxy that are typically empty: for example, a spatial resolution of $\sim 0.1 \mathrm{pc}$ in the outskirts of a galaxy corresponds to a mass resolution of $\sim 10^{-10}$ $10^{-9}$ solar masses. Instead, we propose to study both Ly $\alpha$ radiative transfer and hydrodynamical behavior on the smallest scales and then to use this knowledge as a subgrid recipe.

\section{Conclusions}

Motivated by several observations and a recent theoretical work by McCourt et al. (2016), we studied Ly $\alpha$ radiative transfer in an extremely clumpy medium, i.e., with large number of clumps per 
sightline (up to $f_{\mathrm{c}} \sim 1000$ ). Our main findings on Ly $\alpha$ radiative transfer through clumpy media are

- The behavior of a multiphase medium depends strongly on the "clumpiness" of the system even when keeping the other parameters, such as the total column density, constant.

- In particular, we identify a threshold above which Ly $\alpha$ photons escape preferentially via frequency excursion, i.e., above which multiphase media affect Ly $\alpha$ as if they were homogeneous. This transition depends on clump column density and can be estimated analytically. We found the threshold for the static case to be

$$
f_{\mathrm{c}, \text { crit }} \approx \begin{cases}\frac{2 \sqrt{a_{v} \tau_{0, \mathrm{cl}}}}{3 \pi^{1 / 4}} & \text { for } \sqrt{3} a_{v} \tau_{0} \gtrsim 19 \\ \frac{1.65}{1-\mathrm{e}^{-\tau_{0, \mathrm{cl}}}} & \text { otherwise. }\end{cases}
$$

- The value of this threshold between a clumpy and homogeneous nature further depends on the clump kinematics in a way that can also be estimated analytically. If the clump motion is uncorrelated and Maxwellian, we find that the threshold is given by

$$
f_{\mathrm{c}, \text { crit }} \approx \max \left(\frac{3 \sigma_{\mathrm{cl}}}{2 v_{\mathrm{th}} \sqrt{\ln \left(4 / 3 \tau_{0, \mathrm{cl}}\right)}}, 1.5\right) \text {. }
$$

This is valid for sufficiently large clump motion, i.e., $\sigma_{\mathrm{cl}} \gtrsim$ $v_{\text {th }}$. For smaller values, the system approaches the static case above. Furthermore, we expect large-scale correlations in velocity the transition between these extreme cases. We will investigate this in a future study.

- A similar threshold was found for outflowing clumps (Sect. 2.3.4). We also showed that for outflowing clumps, increasing $f_{\mathrm{c}}$ naturally leads to more asymmetric line profiles, which is in much better agreement with what has been observed in observations of galaxies.

These results suggest important implications for the interpretation of observed Ly $\alpha$ spectra, as a multiphase medium is physically more motivated than simplified homogeneous geometries such as the "shell model". Nevertheless since shell models successfully reproduce observed spectra, they are frequently used to model observations. Because a medium with sufficiently large covering factor behaves as a homogeneous medium, the success of shell models may indicate large covering factors are typical in galaxies as predicted by McCourt et al. (2016). Specifically, we found typical values of $f_{\mathrm{c} \text {, crit }} \sim 10-50$, which are much smaller than $f_{\mathrm{c}} \gtrsim 1000$ predicted in their work. In this picture, it is easy to understand the convergence to the shell model.

Motivated by these results, we fitted shell models to spectra emerging from extremely clumpy outflows undergoing Hubble flow. We found that the column density from the shell closely matches that of the collection of clumps as a whole and the shell expansion velocity appears to be the mass weighted average velocity. This result is very promising as it suggests that the shell model provides us with a fast method of extracting some physical properties of the interstellar and circumgalactic medium from the Ly $\alpha$ spectral line shape. In addition, the value of other shell parameters (e.g., intrinsic Ly $\alpha$ line width prior to scattering) should not necessarily be interpreted literally as physical. We will explore this systematically in future work.

Another implication concerns the mismatch between observed Ly $\alpha$ spectra and those predicted by theoretical studies of
Ly $\alpha$ radiative transfer utilizing hydrodynamical simulations for their input geometry. Our work suggests that this mismatch can be due to the existence of tiny clumps in the observed systems that cannot form even in the most modern hydrodynamical simulations of galaxies because of their limited resolution. Thus, setups of these simulations might yield effective covering factors that are too low, causing the spectra to possess, e.g., a flux at line center that is too large. We will use our results for radiative transfer on small scales to develop an effective theory that can be implemented as a subgrid model in global simulations of galaxies.

Acknowledgements. This research made use of NASA's Astrophysics Data System, and a number of open source software such as the IPython package (Pérez \& Granger 2007); matplotlib (Hunter 2007); and SciPy (Jones et al. 2001). M.D. and M.G. thank the astronomy group at UCSB for their hospitality, and the organizers and participants of "SnowCLAW 2017" for an inspiring conference. M.M. was supported by NASA grant HST-HF2-51376.001A, under NASA contract NAS5-26555. S.P.O. acknowledges NASA grant NNX15AK81G.

\section{References}

Adams, T. F. 1972, ApJ, 174, 439

Adams, T. F. 1975, ApJ, 201, 350

Ahn, S.-H., Lee, H., \& Lee, H. M. 2002, ApJ, 567, 922

Ahn, S.-H., Lee, H.-W., \& Lee, H. M. 2003, MNRAS, 340, 863

Arav, N., Barlow, T. A., Laor, A., \& Blandford, R. D. 1997, MNRAS, 288, 1015

Auer, L. H. P. U. O. 1968, ApJ, 153, 783

Bacon, R., Accardo, M., Adjali, L., et al. 2010, in Ground-based and Airborne Instrumentation for Astronomy III, Proc. SPIE, 7735, 773508

Barnes, L. A., Haehnelt, M. G., Tescari, E., \& Viel, M. 2011, MNRAS, 416, 1723

Barnes, L. A., Garel, T., \& Kacprzak, G. G. 2014, PASP, 126, 969

Behrens, C., \& Braun, H. 2014, A\&A, 572, A74

Blanc, G. A., Adams, J. J., Gebhardt, K., et al. 2011, ApJ, 736, 31

Bonilha, J. R. M., Ferch, R., Salpeter, E. E., Slater, G., \& Noerdlinger, P. D. 1979, ApJ, 233, 649

Cai, Z., Fan, X., Yang, Y., et al. 2017, ApJ, 837, 71

Cantalupo, S., Arrigoni-Battaia, F., Prochaska, J. X., Hennawi, J. F., \& Madau, P. 2014, Nature, 506, 63

Dijkstra, M. 2014, PASA, 31, 26

Dijkstra, M., \& Jeeson-Daniel, A. 2013, MNRAS, 435, 3333

Dijkstra, M., \& Kramer, R. 2012, MNRAS, 424, 1672

Dijkstra, M., Haiman, Z., \& Spaans, M. 2006, ApJ, 649, 14

Dijkstra, M., Wyithe, J. S. B., \& Haiman, Z. 2007, MNRAS, 379, 253

Dijkstra, M., Gronke, M., \& Venkatesan, A. 2016, ApJ, 828, 71

Duval, F., Schaerer, D., Östlin, G., \& Laursen, P. 2014, A\&A, 562, A52

Erb, D. K., Steidel, C. C., Trainor, R. F., et al. 2014, ApJ, 795, 33

Faucher-Giguère, C.-A., Hopkins, P. F., Kereš, D., et al. 2015, MNRAS, 449, 987

Gronke, M., \& Dijkstra, M. 2014, MNRAS, 1103, 10

Gronke, M., \& Dijkstra, M. 2016, ApJ, 826, 14

Gronke, M., Bull, P., \& Dijkstra, M. 2015, ApJ, 812, 123

Gronke, M., Dijkstra, M., McCourt, M., \& Oh, S. P. 2016, ApJ, 833, L26

Hansen, M., \& Oh, S. P. 2006, MNRAS, 367, 979

Harrington, J. P. 1973, MNRAS, 162, 43

Hashimoto, T., Verhamme, A., Ouchi, M., et al. 2015, ApJ, 812, 157

Hayes, M. 2015, PASA, 32, e027

Hayes, M., Schaerer, D., Östlin, G., et al. 2011, ApJ, 730, 8

Hennawi, J. F., Prochaska, J. X., Cantalupo, S., \& Arrigoni-Battaia, F. 2015, Science, 348, 779

Henry, A., Scarlata, C., Martin, C. L., \& Erb, D. 2015, ApJ, 809, 19

Herenz, E. C., Gruyters, P., Orlitova, I., et al. 2016, A\&A, 587, A78

Hogan, C. J., \& Weymann, R. J. 1987, MNRAS, 225, 1P

Hunter, J. D. 2007, Comput. Sci. Engin., 9, 90

Jones, E., Oliphant, T., Peterson, P., et al. 2001, SciPy: Open source scientific tools for Python (Princeton University Press)

Karman, W., Caputi, K. I., Caminha, G. B., et al. 2017, A\&A, 599, A28

Kulas, K. R., Shapley, A. E., Kollmeier, J. A., et al. 2012, ApJ, 745, 33

Laursen, P., \& Sommer-Larsen, J. 2007, ApJ, 657, L69

Laursen, P., Sommer-Larsen, J., \& Andersen, A. C. 2009, ApJ, 704, 1640

Laursen, P., Sommer-Larsen, J., \& Razoumov, A. O. 2011, ApJ, 728, 52

Laursen, P., Duval, F., \& Östlin, G. 2013, ApJ, 766, 124 
Liang, C. J., Kravtsov, A. V., \& Agertz, O. 2016, MNRAS, 458, 1164

Mas-Ribas, L., \& Dijkstra, M. 2016, ApJ, 822, 84

McCourt, M., Oh, S. P., O’Leary, R. M., \& Madigan, A.-M. 2016, ArXiv e-prints [arXiv: 1610.01164]

McKee, C. F., \& Ostriker, J. P. 1977, ApJ, 218, 148

Neufeld, D. A. 1990, ApJ, 350, 216

Neufeld, D. A. 1991, ApJ, 370, L85

Osterbrock, D. E. 1962, ApJ, 135, 195

Östlin, G., Hayes, M., Duval, F., et al. 2014, ApJ, 797, 11

Pei, Y. C. 1992, ApJ, 395, 130

Pérez, F., \& Granger, B. E. 2007, Comput. Sci. Engin., 9, 21

Rauch, M., Sargent, W. L. W., \& Barlow, T. A. 1999, ApJ, 515, 500

Rees, M. J. 1987, MNRAS, 228, 47

Rivera-Thorsen, T. E., Hayes, M., Östlin, G., et al. 2015, ApJ, 805, 14

Smith, A., Safranek-Shrader, C., Bromm, V., \& Milosavljevi, M. 2015, MNRAS, 449,4336
Song, M., Finkelstein, S. L., Gebhardt, K., et al. 2014, ApJ, 791, 3 Steidel, C. C., Erb, D. K., Shapley, A. E., et al. 2010, ApJ, 717, 289 Steidel, C. C., Rudie, G. C., Strom, A. L., et al. 2014, ApJ, 795, 165 Tasitsiomi, A. 2006, ApJ, 645, 792

Trainor, R. F., Steidel, C. C., Strom, A. L., \& Rudie, G. C. 2015, ApJ, 809, 89

Trebitsch, M., Verhamme, A., Blaizot, J., \& Rosdahl, J. 2016, A\&A, 593, A122

Verhamme, A., Schaerer, D., \& Maselli, A. 2006, A\&A, 460, 397 Verhamme, A., Dubois, Y., Blaizot, J., et al. 2012, A\&A, 546, A111

Verhamme, A., Orlitová, I., Schaerer, D., \& Hayes, M. 2015, A\&A, 578, A7

Yamada, T., Matsuda, Y., Kousai, K., et al. 2012, ApJ, 751, 29

Yang, H., Malhotra, S., Gronke, M., et al. 2016, ApJ, 820, 130

Yang, H., Malhotra, S., Gronke, M., et al. 2017, ApJ, 844, 171

Zheng, Z., \& Miralda-Escudé, J. 2002, ApJ, 578, 33

Zheng, Z., Cen, R., Trac, H., \& Miralda-Escudé, J. 2010, ApJ, 716, 574 


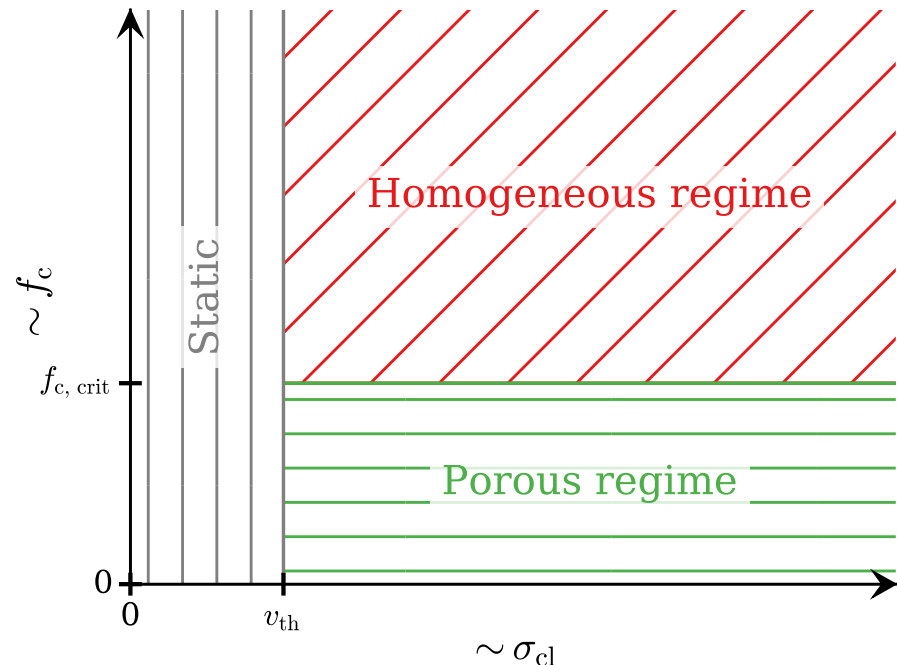

Fig. A.1. Escape regimes of a medium with (uncorrelated) randomly moving clumps as discussed in Appendix A.

\section{Appendix A: Regimes of a medium with uncorrelated clump motion}

For randomly moving, optically thick clumps the photons either escape through holes in velocity space (if $f_{\mathrm{c}} \lesssim f_{\text {c, crit }}$, Eq. (12)) or escape in single flight (for $f_{\mathrm{c}} \gtrsim f_{\mathrm{c} \text {, crit }}$ ), as described in Sect. 2.3.4. In the former case, the emergent spectrum is similar to the intrinsic spectrum, that is, narrow and single peaked. If, however, $f_{\mathrm{c}}>f_{\mathrm{c} \text {, crit }}$ the radiative transfer process is similar to a slab with temperature $T_{\text {eff }}$ (Eq. (11)), which means the photons escape in a single flight after interaction with one (fast-moving) clump, and so will the emergent double-peaked spectrum, i.e., a peak position of $x_{\mathrm{p}} \sim x_{*}$ or in (observed) velocity units

$v_{\mathrm{p}} \approx x_{*} v_{\mathrm{th}}\left(T_{\mathrm{eff}}\right) \approx 3.8 \sigma_{\mathrm{cl}}$.

Figure A.1 shows a visual overview of these regimes. In this figure, we also indicated that a smaller clump motion than the internal thermal motion of the atoms (for the parameters used in this work of $\sigma_{\mathrm{cl}} \lesssim 13 \mathrm{~km} \mathrm{~s}^{-1}$ ) leads to a convergence back to the static case.

Another interesting part of the parameter space is between the two regimes, for $f_{\mathrm{c}} \sim f_{\mathrm{c} \text {, crit }}$. Here, the velocity space is sufficiently sampled so that hardly any photons can escape without clump interaction. However, after an interaction with a (slowly moving) clump the probability of interacting with another clump is small - even if the photon is still in the core of the line. This is because the velocity distribution is not that well sampled to provide $\tau_{0} \gg 1$. As a result, the emergent spectrum directly represents the clumps' velocity dispersion of the clumps, which means a single-peaked spectrum a line center of width $\sim \sigma_{\mathrm{cl}}$.

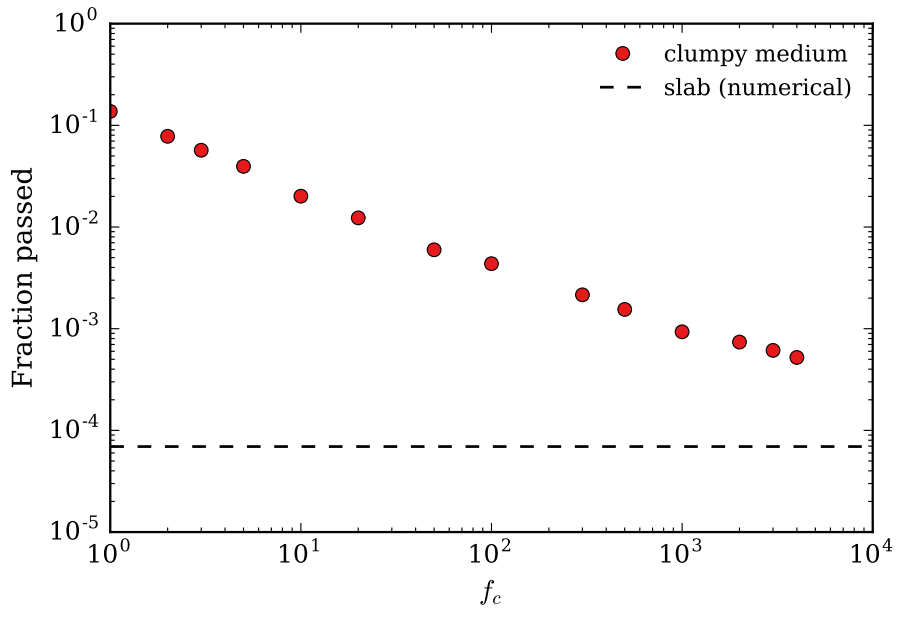

Fig. B.1. Transmission through a clumpy slab with column density $N_{\mathrm{HI}, \text { total }}=\frac{4}{3} \times 10^{19} \mathrm{~cm}^{-2}$ (as before, measured per half-height) vs. covering factor $f_{\mathrm{c}}$. In comparison we show the transmission through a (solid) slab with the same column density.

In summary, with increasing covering factor, a medium with uncorrelated clump motion can lead to a narrow or wide singlepeaked spectrum (of widths of the intrinsic spectrum or clump velocity dispersion, respectively) at line center or a wide doublepeaked spectrum (if $f_{\mathrm{c}} \gtrsim f_{\mathrm{c} \text {, crit }}$ ).

\section{Appendix B: Additional numerical results}

\section{B.1. Transmission through a clumpy slab}

Figure B.1 shows the fraction of photons that passed through a clumpy medium when emitted at the boundary of the box for a fixed total column density but various number of clumps per sightline. The transmitted fraction of photons decreases with increasing covering factor and approaches the limit of a homogeneous slab. We attribute this dependence on $f_{\mathrm{c}}$ and the differences compared to a homogeneous medium to surface effects, i.e., because of the roughness of the boundary it is easier for photons to get trapped in the slab.

\section{B.2. Examples of photon trajectories}

Figure B.2 shows examples for the three different escape mechanisms discussed in this work. The left panel shows a random walk in a static medium, the central panel shows escape through excursion, and the right panel shows the escape through single flight. An animated version of Fig. B. 2 is available online ${ }^{10}$. 


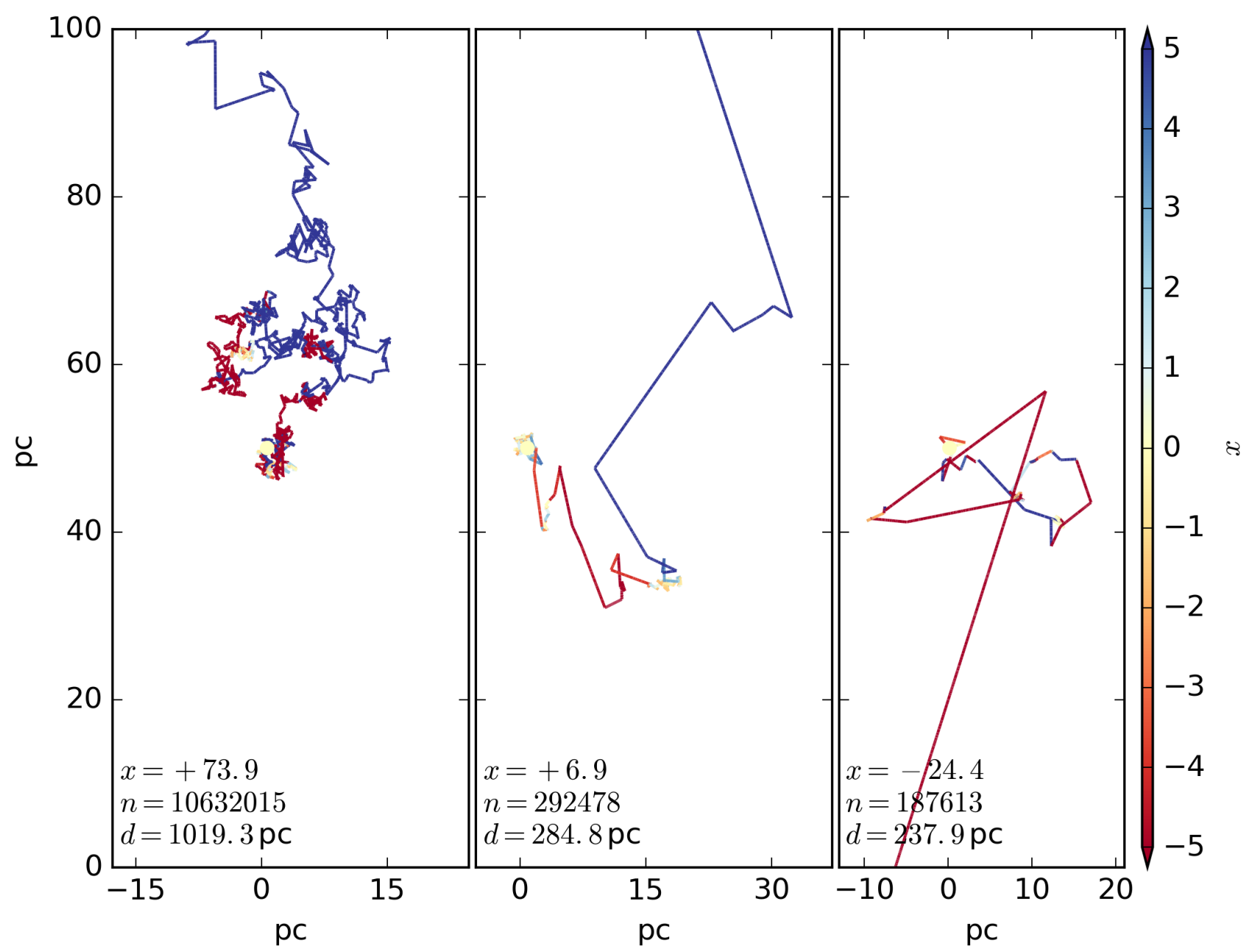

Fig. B.2. Examples of photon trajectories. The left panel shows a photon escaping through the random walk from a static medium with $\left(N_{\mathrm{HI}, \mathrm{cl}}, f_{\mathrm{c}}\right)=$ $\left(10^{20} \mathrm{~cm}^{-2}, 100\right)$. In the central panel the photon escapes in an excursion $\left[\left(N_{\mathrm{HI}, \mathrm{cl}}, f_{\mathrm{c}}\right)=\left(10^{17} \mathrm{~cm}^{-2}, 100\right)\right]$ after a random walk and in the right panel nearly directly through excursion/single flight due to movement of the clumps $\left[\left(N_{\mathrm{HI}, \mathrm{cl}}, f_{\mathrm{c}}, \sigma_{\mathrm{cl}}\right)=\left(10^{17} \mathrm{~cm}^{-2}, 100,100 \mathrm{~km} \mathrm{~s}{ }^{-1}\right)\right]$. In each panel, the escape frequency $x$ is shown along with the total number of scatterings $n$, and the distance traveled $d$. In addition, the color coding represents the frequency of the photon (truncated at \pm 5 ). An animated version of this figure is available online. 